\title{
Application of causality analysis on nuclear reactor systems 루
}

\author{
Cite as: Chaos 29, 043126 (2019); doi: 10.1063/1.5083905 \\ Submitted: 1 December 2018 . Accepted: 3 April 2019. \\ Published Online: 24 April 2019

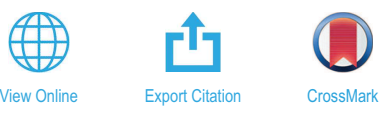

D. Chionis, ${ }^{1,2, a)}$ A. Dokhane, ${ }^{1, \text { a) }}$ H. Ferroukhi, ${ }^{1}$ and A. Pautz ${ }^{2,3}$

\begin{abstract}
AFFILIATIONS
${ }^{1}$ Paul Scherrer Institut, Laboratory for Reactor Physics and Thermal-Hydraulics, CH-5232 Villigen PSI, Switzerland

${ }^{2}$ Swiss Federal Institute of Technology in Lausanne, CH-1015 Lausanne, Switzerland

${ }^{3}$ Paul Scherrer Institut, Nuclear Energy and Safety Division, CH-5232 Villigen PSI, Switzerland
\end{abstract}

${ }^{a}$ Authors to whom correspondence should be addressed: dionysios.chionis@psi.ch and abdelhamid.dokhane@psi.ch

\begin{abstract}
Causality analysis is a substantial tool for identifying cause-and-effect links between different components of a system and has been extensively used in various areas of science such as neuroscience, climatology, and econometrics. This analysis is carried out in terms of the renormalized partial directed coherence and the directed transfer function connectivity measures. Applying such analysis in the nuclear reactor field is of paramount importance since it can help in inferring cause-and-effect relationships between highly coupled processes, and consequently, it can assist on the safe and reliable operation of a nuclear power plant during the occurrence of possible disturbances or malfunctions. The effectiveness of the connectivity analysis is demonstrated through several simulated and measured test cases. Results show that the connectivity analysis is able to identify accurately the importance and central role of the activation signal when it is applied on a simple analytical model and a simulated nuclear reactor system. In addition, the application on more realistic and complex measured data sets of a Swiss boiling water reactor illustrates the capability of this analysis to indicate possible causes behind the observed anomalies or trends observed at certain conditions and, more importantly, allows a better understanding of the underlying interactions among different neutronic and thermal-hydraulic processes.
\end{abstract}

Published under license by AIP Publishing. https://doi.org/10.1063/1.5083905

Causality analysis is commonly used for identifying the causeand-effect relationships between various signals within multivariate and complex models. The signals' interconnectivity patterns have already been successfully estimated in the fields of econometrics, climatology, and neuroscience. This paper is the first systematic approach for applying causality analysis techniques on nuclear reactor systems. To this aim, the most prominent causality measures have been implemented in the Paul Scherrer Institute (PSI) connectivity methodology and both simulated and measured plant data have been analyzed. The present paper demonstrates the capability of the connectivity methodology to infer the causal interactions between the nuclear reactor signals and to indicate the root cause of simulated disturbances or measured anomalies. Most importantly, the connectivity methodology can assist the nuclear reactor operators and the nuclear research to deeper and better understand the underlying mechanisms of a nuclear reactor.

\section{INTRODUCTION}

Time-series analysis methods are commonly used in many research areas for extracting useful information related to the structure and the characteristics of the analyzed system. Inferring the underlying interconnections between signals of complex systems, such as the human brain, social networks, stock markets, etc., is a challenging and complicated task.

Similar developments and applications of time-series analysis techniques for monitoring and diagnostics of the nuclear reactors' operation continue to be a hot topic of research. ${ }^{1}$ These techniques provide an invaluable source of information for gaining better insights into the underlying processes, usually analyzed in terms of coherence analysis for estimating the level of correlation between signals in the frequency domain. However, nuclear reactors are complex systems susceptible to operational problems due to the appearance of anomalies and disturbances. Therefore, one crucial challenge is to characterize the type and the source of a given anomaly/disturbance 
in order to perform all the proper actions which will ensure the continuation of the safe and reliable operation of the nuclear power plant. Unfortunately, the coherence function, which can be applied only on a pair of signals (bivariate systems), cannot provide any information about either the direction of information flow or the root cause of the disturbance.

During the last few decades, causality analysis techniques have been developed for the identification of the cause-and-effect relationships between analyzed signals, offering valuable information about the structural behavior of the examined system. Among these techniques, advanced connectivity measures, based on the mathematical formulation of the so-called Granger causality, have been developed and successfully and extensively applied in the field of neuroscience. The partial directed coherence (PDC) is a powerful connectivity measure, and its renormalized version (i.e., the rPDC) has been utilized not only for evaluating the direct causal interactions between various signals but also for estimating the strength of their interconnections. Furthermore, the directed transfer function (DTF) is another useful connectivity measure, which can be used for analyzing the propagation of information within multivariate systems. Even though DTF cannot distinguish between direct and indirect interconnections (as the case for PDC and rPDC), it is reflecting the signals reachability and, therefore, offers valuable information in the understanding of the structural network of the analyzed system.

Benefiting from these advances in causality analysis, the main goal of the current paper is to apply the principles of connectivity analysis on nuclear reactor data in order to extract valuable information about the connectivity patterns between the various simultaneously recorded signals during malfunctioning operations induced by potential anomalies and/or disturbances. At the same time, the application of these techniques on highly coupled nuclear reactor systems points out some limitations of the causality analysis. To the authors' knowledge, this is the first systematic application of such techniques in the nuclear reactor field.

This paper is organized as follows: First, the formulations of coherence function and the PDC, rPDC, and DTF connectivity measures are briefly described. After that, the connectivity analysis methodology, as it is applied in nuclear engineering applications at PSI, is elaborated in Sec. II. A simple numerical example is used then in Sec. III for illustrating the advantages and drawbacks of the utilized connectivity measures. Then, a perturbation on a simulated reactor system is examined and the source of disturbance is estimated in Sec. IV. Section V presents the connectivity analysis results on real nuclear power plant data. Finally, the conclusions are presented in Sec. VI.

\section{METHODOLOGY}

Traditional signal analysis techniques are estimating the similarity between two processes by using the correlation and coherence functions in the time and frequency domain, respectively. Both functions provide information about the coupling between two signals without requiring advanced signal processing techniques. Processes that are rich in oscillatory content are typically analyzed in the frequency domain, and the coherence function is utilized for estimating common shared spectral components between the analyzed signals. The coherence function between two simultaneously measured signals $y_{i}$ and $y_{j}$ is estimated in the frequency domain as

$$
\operatorname{Coh}_{i j}(f)=\frac{\left|P_{i j}(f)\right|^{2}}{P_{i i}(f) P_{j j}(f)},
$$

where $P_{i i}$ and $P_{j j}$ are the auto-spectral density functions of signals $y_{i}$ and $y_{j}$, respectively, and $P_{i j}$ corresponds to the signals' cross-spectral density function. Coherence is a complex number, characterized by its phase and modulus. The first component indicates the phase difference between the analyzed signals, whereas the second component corresponds to the signals' connection strength in the frequency domain. ${ }^{2}$ Due to the normalization scheme used in (1), the coherence function lies between 0 (absence of correlation) and 1 (full correlation/synchronization). Coherence is a powerful tool in connectivity analysis as it can estimate very fast and without a lot of computation power whether there is a strong coupling between two signals by quantifying the strength of the link between their oscillatory components. ${ }^{3}$ However, the coherence function has a reciprocal behavior [i.e., $\left.\operatorname{Coh}_{i j}(f)=\operatorname{Coh}_{j i}(f)\right]$ and can be defined only for bivariate systems (i.e., system with only two signals). In other words, the coherence function cannot be used in order to identify the direction of an interaction (i.e., whether signal $y_{i}$ is the driving force of another signal $y_{j}$ or vice versa), or for estimating if two signals are connected between each other either directly or a third signal is interfering between them (indirect interaction). Therefore, the analysis of complex multivariate systems becomes impossible by using the traditional coherence function.

In order to overcome the coherence function's limitations, as described previously, new methods in connectivity analysis have been introduced for identifying cause-and-effect relationships between signals in multivariate systems. Most of these techniques have been related at some extent to the concept of Granger causality which was introduced back in $1969 .{ }^{4}$ Granger introduced the concept of causality in the field of econometrics stating that if the past of a time series $y$ improves the prediction of the future of a second simultaneously recorded time series $x$, then the former is said to cause the latter. Granger causality was developed in the time domain and implemented first on bivariate and three-variate systems, and then it was generalized for multivariate systems. ${ }^{4}$ Extensions in the frequency domain and a multivariate formulation have been further proposed in the literature. ${ }^{5,6}$ Most importantly, Granger causality is an unreciprocal expression, i.e., if a signal $y$ is causal to signal $x$ then that does not necessarily imply that $x$ is causal to $y$. Some of the most prominent causality measures are the PDC, rPDC, and DTF measures.

The accurate evaluation of the PDC/rPDC/DTF connectivity measures depends on the adequate representation of the analyzed system through a multivariate autoregressive model (MVAR). For a set of $m$ simultaneously recorded signals $y(t)=\left[y_{1}(t), y_{2}(t)\right.$, $\left.\ldots, y_{m}(t)\right]^{\prime}$ in the time domain, a MVAR model of order $p$ is expressed as

$$
\boldsymbol{y}(t)=\sum_{k=1}^{p} \boldsymbol{\alpha}(k) \boldsymbol{y}(t-k)+\boldsymbol{\varepsilon}(t),
$$

where $\boldsymbol{\alpha}(k)$ is the MVAR model coefficient matrix of size [ $m \times m \times p]$ for which the coefficients $\boldsymbol{\alpha}_{i j}(k)$ describe the linear influence of signal $y_{j}(t)$ on signal $y_{i}(t-k)$, and $\boldsymbol{\varepsilon}(t)$ is a multivariate Gaussian white 
noise vector. In other words, Eq. (2) expresses the present value of each signal $y_{i}(t)$ as a function of the $p$ past time steps of all simultaneously recorded signals. The model coefficients $\boldsymbol{\alpha}(k)$ express the causal relationships between the analyzed signals in the time domain. Therefore, it can be said that a signal $y_{j}$ affects the behavior of a signal $y_{i}$ if and only if the respective model coefficient $\boldsymbol{\alpha}_{i j}$ is non-zero for at least one time lag $k$. The accurate representation of the underlying causal interactions through the model coefficients $\boldsymbol{\alpha}(k)$ depends on the MVAR model properties. Therefore, long enough stationary signals (several data points more than the total estimated modal parameters $m p^{2}$ ) shall be detrended (subtracting trends and mean values) and standardized (normalized over the signal's standard deviation) before an algorithm for estimating the best-estimate model order $p$ is used. ${ }^{2}$ The PSI connectivity analysis methodology includes several model order selection algorithms (e.g., Akaike information criterion, Bayesian information criterion, Hannan-Quinn criterion, etc. ${ }^{7}$ ) which have been extensively used in the literature and intent to optimally balance between under-fitting and overfitting the analyzed data set. The criterion which estimates the minimum model $p$ order among the available algorithms is the one to be selected in this work depending on the analyzed scenario presented in Secs. III-V. Based on the evaluated model order $p$, the data set $y(t)$ is fitted to a MVAR model using the robust and best-estimate Nuttal-Strand algorithm by minimizing the prediction error. ${ }^{6-8}$ It is important to note that the interpretation of the results of connectivity measures is not always a straightforward task. It has been demonstrated in the literature that various factors (e.g., signals' noise, low measurement resolution, missing root cause signals from the analyzed system, etc.) can affect the connectivity results and spurious interconnections can be estimated leading to false identification of the cause-and-effect relationships. ${ }^{2,9,10}$ Therefore, a detailed and systematic postprocessing of the measured signals is needed in order to identify the key processes of the studied system, which minimizes the risk of the erroneous interpretation of the connectivity results.

\section{A. Partial directed coherence (PDC)}

The partial directed coherence (PDC) connectivity measure has been proposed by Ref. 11 and has been widely applied in neuroscience, econometrics, weather forecasting, etc. PDC aims at the estimation of the direct directional interactions between simultaneously recorded signals. Its formulation is derived from the Fourier transformation of the MVAR model coefficients $\boldsymbol{\alpha}(k)$

$$
\boldsymbol{A}_{i j}(f)=\boldsymbol{I}-\sum_{r=1}^{p} \boldsymbol{\alpha}_{i j}(r) e^{-2 \pi f r \sqrt{-1}},
$$

where $\boldsymbol{I}$ is the $[m \times m]$ identity matrix. The PDC connectivity measure is then defined as

$$
\operatorname{PDC}_{i j}(f)=\frac{\left|\boldsymbol{A}_{i j}(f)\right|}{\sqrt{\sum_{q=1}^{m}\left|\boldsymbol{A}_{q j}(f)\right|^{2}}} .
$$

PDC is normalized in $[0,1]$, and it has the main advantage to differentiate the direct from the indirect interconnections within the analyzed multivariate system. Since the denominator of Eq. (4) accounts for the total outflow information from signal $y_{j}$ to all the other signals, PDC indicates the relative strength of the direct effect of a signal $y_{j}$ on $y_{i}$ as compared to the strength of the direct links of $y_{j}$ to all the other processes of the system. The critical value of the PDC measure, which estimates whether or not a direct interconnection is statistically significant at the $\theta$-significance level of $5 \%$ (rejection of null hypothesis), is estimated based on the statistical properties that have been introduced in Ref. 12.

\section{B. Renormalized partial directed coherence (rPDC)}

PDC is a key causality measure, which significantly improves the understanding of the underlying connectivity mechanisms of a system. However, due to the normalization scheme of Eq. (4), PDC measures the strength of direct influences relative to a given signal source and, therefore, it cannot give clear conclusions when direct connectivity strengths are compared at different frequencies or between different pairs of signals within the system. In other words, higher PDC values between a signal pair do not imply undoubtedly stronger connection link compared to another pair of signals. ${ }^{13}$ In order to overcome that limitation, the rPDC measure has been proposed not only for estimating the direct causal interactions (as PDC does) but also for evaluating the connections' strength within the analyzed multivariate system.

To introduce the rPDC measure, the two-dimensional vector $Z_{i j}(f)$ is defined, which consists of the real and imaginary parts of the Fourier transformed coefficients $\boldsymbol{A}_{i j}(f)$ [as defined in Eq. (3)]. The corresponding estimator $\hat{\boldsymbol{Z}}_{i j}(f)$ is asymptotically Gaussian distributed with mean value $\boldsymbol{Z}_{i j}(f)$ and covariance matrix $\boldsymbol{\Sigma}_{\hat{Z}}$ defined as

$$
\begin{aligned}
\boldsymbol{\Sigma}_{\hat{z}}= & \frac{\boldsymbol{V}_{i j}(f)}{N_{m}}=\sum_{r, l=1}^{p} \boldsymbol{R}_{j j}^{-1}(r, l) \boldsymbol{\Sigma}_{i i} \\
& \times \frac{1}{\mathrm{~N}_{m}}\left[\begin{array}{ll}
\cos (r f) \cos (l f) & \cos (r f) \sin (l f) \\
\sin (r f) \cos (l f) & \sin (r f) \sin (l f)
\end{array}\right],
\end{aligned}
$$

where $N_{m}$ is the number of data points, $\boldsymbol{R}$ is the covariance matrix of the MVAR model [based on Eq. (2)], and $\boldsymbol{\Sigma}$ is the covariance matrix of the noise process. Based on the covariance matrix estimator $\boldsymbol{\Sigma}_{\hat{Z}}$ [Eq. (5)], the rPDC measure is then defined as

$$
r P D C_{i j}(f)=\boldsymbol{Z}_{i j}(f)^{\prime} \boldsymbol{V}_{i j}^{-1}(f) \boldsymbol{Z}_{i j}(f) .
$$

Similarly, to the definition of the PDC measure, the rPDC is not reciprocal; in other words, signal $y_{j}$ may directly affect $y_{i}$ without necessarily $y_{i}$ causing $y_{j}$. If $r P D C_{i j}(f)=0$, then the null hypothesis is true and a direct Granger-causal influence from signal $y_{j}$ to signal $y_{i}$ can be rejected at a frequency $f$. The critical value for a $\theta$-significance level for rejecting the null hypothesis is given by $\chi^{2}{ }_{2,1-\theta} / N_{m}$, where $\chi_{2,1-\theta}^{2}$ indicates the 1- $\theta$ quantile of the chi-square distribution with two degrees of freedom, as it is introduced in Ref. 13.

\section{Directed transfer function (DTF)}

The DTF measure was introduced in Ref. 14 in order to describe the causal influence of channel $y_{j}$ on channel $y_{i}$ by reflecting the signals reachability within the analyzed multivariate system. ${ }^{15}$ The DTF measure has a similar formulation as PDC, and it is based on the 
TABLE I. Properties of frequency domain connectivity measures.

\begin{tabular}{|c|c|c|c|c|c|c|}
\hline $\begin{array}{l}\text { Connectivity } \\
\text { measure }\end{array}$ & Bivariate & Multivariate & Undirected & Directed & Indirect & Direct \\
\hline Coherence & $\mathrm{x}$ & & $\mathrm{x}$ & & & \\
\hline PDC & & $\mathrm{x}$ & & $\mathrm{x}$ & & $\mathrm{x}$ \\
\hline $\mathrm{rPDC}$ & & $\mathrm{x}$ & & $\mathrm{x}$ & & $\mathrm{x}$ \\
\hline DTF & & $\mathrm{x}$ & & $\mathrm{x}$ & $\mathrm{x}$ & \\
\hline
\end{tabular}

Fourier transformation of the MVAR model coefficients [Eq. (3)]

$$
\operatorname{DTF}_{i j}(f)=\frac{\left|\boldsymbol{H}_{i j}(f)\right|}{\sqrt{\sum_{q=1}^{m}\left|\boldsymbol{H}_{i q}(f)\right|^{2}}},
$$

where $\boldsymbol{H}_{i j}(f)=\boldsymbol{A}_{i j}(f)^{-1}$ is the transfer function of the MVAR model. DTF is normalized in $[0,1]$ and a directional connection from signal $y_{j}$ to signal $y_{i}$ at frequency $f$ exist if and only if $D T F_{i j}(f) \neq 0$ (rejection of null hypothesis). DTF is a powerful connectivity measure, which estimates the dependencies between the analyzed components in a multivariate system by summing up the direct and indirect influences and consequently provides the direction of information flow.

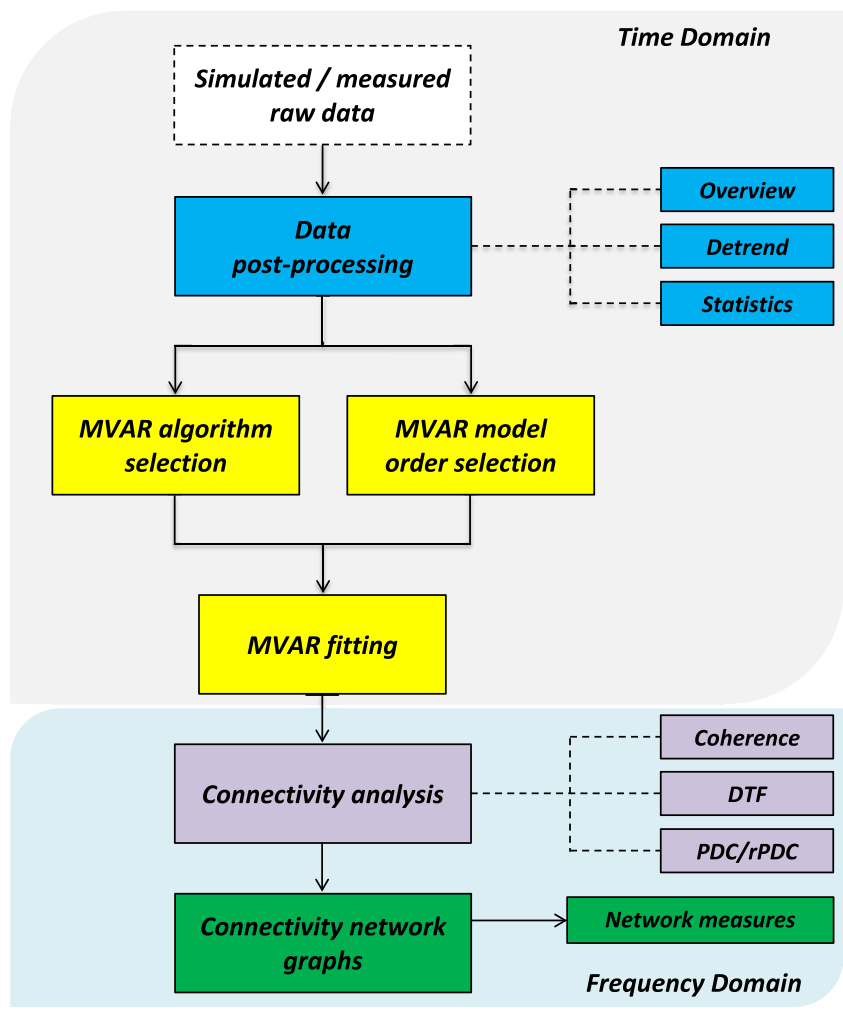

FIG. 1. Connectivity analysis methodology used at PSI.
In this work, the statistical properties of the DTF connectivity measure at $5 \%$ significance level (rejecting the null hypothesis) are used for estimating the statistical significance of DTF values. ${ }^{1}$

Table I summarizes the properties of the four connectivity measures, as described in Subsections II A-II C. The advantages and drawbacks of these connectivity measures are demonstrated in Sec. III, through a simple analytical model in which the connectivity structure and the signal interconnections are well defined. Then, the connectivity analysis is focusing mainly on the rPDC and DTF measures for examining the behavior of simulated and measured nuclear reactor systems in Secs. IV and V, respectively.

\section{Connectivity graphs}

The connectivity analysis results are commonly summarized in terms of connectivity graphs (or path diagrams) following the principles of the graph theory. ${ }^{17}$ To this aim, a signal (e.g., neutron detector response, process signal, etc.) is considered as a node, called also vertex, and the connections as edges. Therefore, any analyzed system can be expressed as a graph $G$ consisting of $N$ nodes and $E$ edges joining the nodes [abbreviated as $G=(N, E)]$. A graph can be classified based on its directionality (i.e., directed-digraph or undirected), and based on its weighs (i.e., weighted or unweighted-binary). Connectivity graphs are used not only for the visualization of the connectivity analysis results, but also for estimating the network measures and the topological characteristics which offer a further understanding of the signals' significance within the analyzed system. This work is focusing on identifying the source(s) that might be responsible for a perturbation on a given simulated or measured system. To this aim, the network measures, used in this analysis, are the "node degree" and the "node strength." The node degree is the simplest and the most fundamental measure, and it estimates the number of connections that one node has within the analyzed system. The node degree is subdivided into in-degree and out-degree, in the case of digraphs such as rPDC, PDC, and DTF, indicating the number of incoming and outgoing connections with respect to a node, respectively. The node strength is used in weighted graphs for estimating the total weights that a node has within the network and is subdivided into in-strength and out-strength, based on the direction of interaction. ${ }^{18}$ Additional information about connectivity graphs and network measures can be found in Refs. 9 and 18.

At PSI, a connectivity analysis methodology has been developed, recently, for studying the information flow paths in nuclear engineering applications by using the coherence function, PDC, rPDC, and DTF connectivity measures. A set of MATLAB scripts [based on the AsympPDC-v.3 (available online via 
http://www.lcs.poli.usp.br/ baccala/pdc/dtf.html) and SIFT toolboxes (available online via https://sccn.ucsd.edu/wiki/SIFT) ${ }^{19}$ ] have been used and appropriately adjusted to nuclear reactors applications. A flow chart summarizing the steps of methodology, based on the description given in Sec. II, is presented in Fig. 1.

\section{ANALYTICAL VERIFICATION}

The importance of the causality measures on identifying the connectivity patterns within a multivariate system is demonstrated through a simple analytical model. Four signals have been generated based on the equation system (8). The activation signal $y_{1}$ is modeled as a damped oscillator with a nominal frequency of $1 \mathrm{~Hz}$. The signals are sampled with a sampling frequency of $8 \mathrm{~Hz}$ over a period of $1250 \mathrm{~s}$ [Fig. 2(a)]. The signal $y_{1}$ is modeled to be the driving force of the analyzed system. The highly oscillatory behavior of all the four signals is illustrated in the time domain in Fig. 2(a). The Fourier transformation of the simulated signals from the time domain to the frequency domain is presented in Fig. 2(b), in which it is observed that all the signals exhibit strong spectral behavior at the nominal frequency of $1 \mathrm{~Hz}$. Predefined direct directional interactions are modeled via the non-zero model coefficients $\alpha_{21}(1), \alpha_{31}(1), \alpha_{42}(1), \alpha_{43}(1)$ for time lag $k=1$ (the values of these coefficients are arbitrary selected and they correspond to the interaction strength from one signal to the other). To this aim, the connectivity analysis is expected to predict direct directional interaction between the signals $y_{1} \rightarrow y_{2}, y_{1} \rightarrow y_{3}, y_{2} \rightarrow y_{4}$, and $y_{3} \rightarrow y_{4}$, respectively. The model order is estimated by the Akaike Information Criterion as $p=2$, and then a $\operatorname{MVAR}(2)$ is fitted to the simulated data

$$
\begin{aligned}
& y_{1}(t)=1.2 y_{1}(t-1)-0.7 y_{1}(t-2)+\varepsilon_{1}(t), \\
& y_{2}(t)=0.5 y_{2}(t-1)-0.3 y_{2}(t-2)-0.3 y_{1}(t-1)+\varepsilon_{2}(t), \\
& y_{3}(t)=0.7 y_{3}(t-1)-0.5 y_{3}(t-2)-0.2 y_{1}(t-1)+\varepsilon_{3}(t), \\
& y_{4}(t)=0.5 y_{4}(t-1)+0.5 y_{2}(t-1)+0.4 y_{3}(t-1)+\varepsilon_{4}(t),
\end{aligned}
$$

a
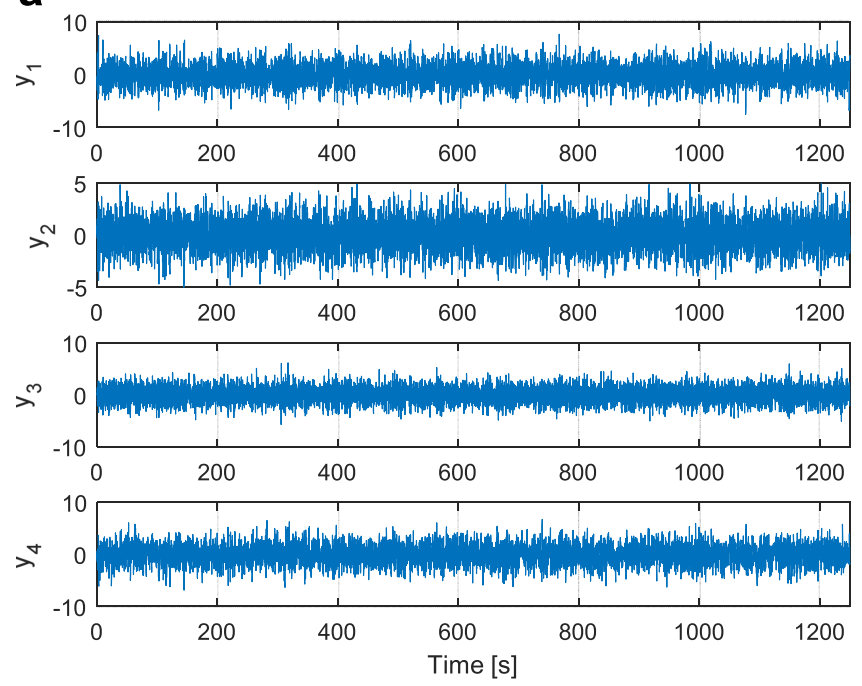

The connectivity analysis results are summarized in four plots in Fig. 3. The connectivity measures of coherence, PDC, rPDC, and DTF are estimated for the simulated system (8) and the results are visualized based on the graph theory properties at the nominal frequency of $1 \mathrm{~Hz}$, following the methodology described in Sec. II. The four signals are depicted as nodes (green vertices) and the connectivity interactions between each other are depicted as weighted edges for all four plots. The coherence function results [Fig. 3(a)] indicate strong undirected interactions between all the four signals. Since the coherence function is applied only in a pair of signals (bivariate analysis), it is impossible to identify the correct connectivity structure between the analyzed signals. Results based on the PDC and the rPDC measures are illustrated in Figs. 3(b) and 3(c), respectively. All the simulated interactions are evaluated correctly by both PDC and rPDC measures, as designed in the analytical model (8). This indicates that the causality measure based on the PDC formulation is detecting properly the direct interactions within the analyzed system, but not their strengths, while the rPDC represents correctly both the direction and the strength. Figure 3(d) shows the connectivity results based on the DTF measure. All the interactions are correctly estimated by the DTF measure likewise in the case of PDC/rPDC measures. However, one additional edge between the signals $y_{1}$ and $y_{4}$ is depicted in Fig. 3(d), since the DTF measure cannot differentiate directly from the indirect interactions, and as stated earlier it represents the summation of the direct and the indirect interactions. This indirect interaction is propagating from $y_{1}$ to $y_{4}$ via the intermediate nodes $y_{2}$ and $y_{3}$. Hence, the property of signals' reachability is reflected by the DTF measure ${ }^{15}$ In other words, the DTF measure is correctly indicating that the information is transferred from the activation signal $y_{1}$ to all the other three signals.

Figure 4 presents the connectivity strength values between the four analyzed signals as a function of the MVAR model coefficients $\boldsymbol{\alpha}_{i j}(t)$ for all four connectivity measures at the nominal

b

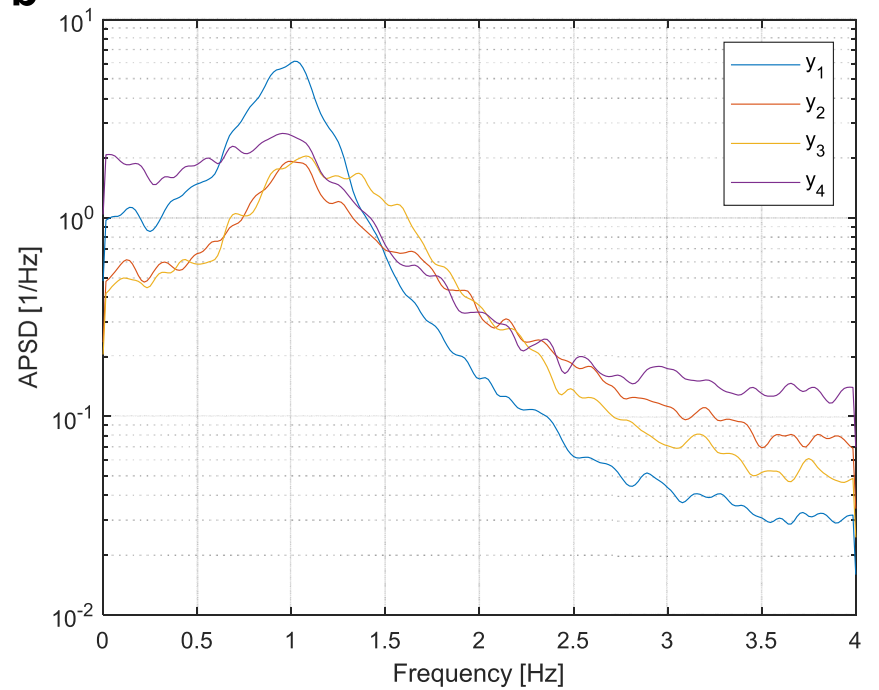

FIG. 2. Simulated signals of the analytical model (8) in the time domain (a) and the frequency-domain (b). 
frequency of $1 \mathrm{~Hz}$. For example, the influence of signal $y_{1}$ to signal $y_{2}$ is modeled via the model coefficient $\left|\alpha_{21}(t-1)\right|=0.3$, as shown in Eq. (8). The connectivity analysis (as summarized in Fig. 3) indicates that the connectivity strengths between processes $y_{1}$ and $y_{2}$ are $P D C_{21}(1 \mathrm{~Hz})=0.6, r P D C_{21}(1 \mathrm{~Hz})=0.1$, $D_{T F}(1 \mathrm{~Hz})=0.8$, and $\operatorname{Coh}_{21}(1 \mathrm{~Hz})=\operatorname{Coh}_{12}(1 \mathrm{~Hz})=0.55$. As a second example, the signals $y_{3}$ and $y_{4}$ are designed to interact via the model coefficient $\left|\alpha_{43}(t-1)\right|=0.4$ [see Eq. (8)], which implies a stronger interconnection compared to that between signals $y_{1}$ and $y_{2}$, as shown before. The connectivity analysis shows that the connectivity strengths between the signals $y_{3}$ and $y_{4}$ are $P D C_{43}(1 \mathrm{~Hz})=0.3, r P D C_{43}(1 \mathrm{~Hz})=0.21, D T F_{43}(1 \mathrm{~Hz})=0.06$, and $\operatorname{Coh}_{43}(1 \mathrm{~Hz})=\mathrm{Coh}_{34}(1 \mathrm{~Hz})=0.18$. Comparing these two examples, it can be clearly observed that higher connectivity strength values of $r P D C$ [i.e., $r P D C_{43}(1 \mathrm{~Hz})>r P D C_{21}(1 \mathrm{~Hz})$ ] correspond to higher model coefficient values of the analyzed system [i.e., $\left.\left|\alpha_{43}(t-1)\right|>\left|\alpha_{21}(t-1)\right|\right]$, as it is depicted also by the red line in Fig. 4 . However, the same statement cannot be made for the other three

a

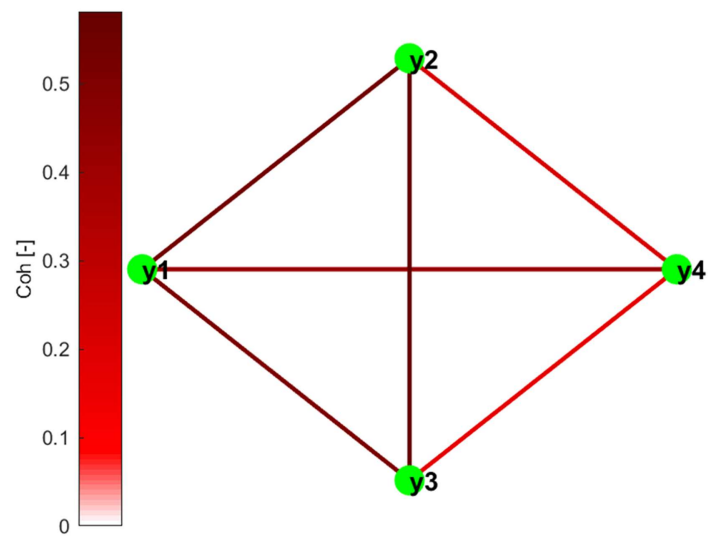

C

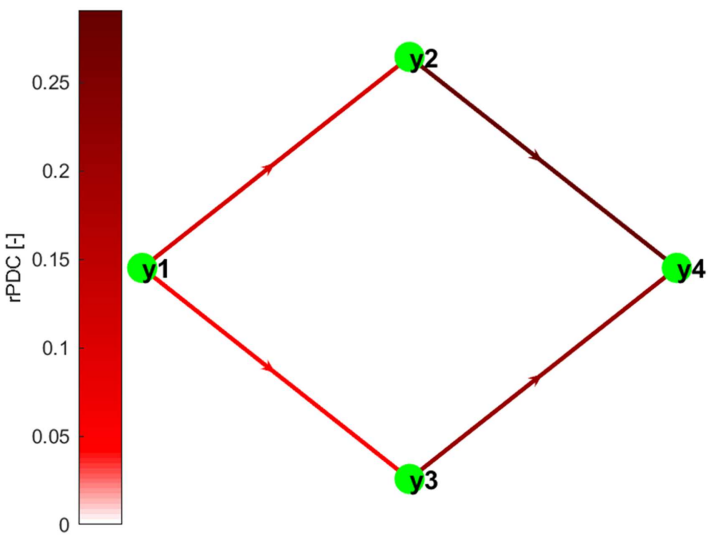

connectivity measures, i.e., PDC, DTF, and Coh, since they are predicting, for some cases, lower connectivity strength results for higher model coefficient values (see Fig. 4). Therefore, the rPDC indicates correctly all the direct directional connections, and more importantly, it is the measure that properly reflects the signals interactions' strength. In other words, the rPDC measure is the best connectivity analysis measure that can be used for understanding deeper the underlying mechanisms between the various processes of an analyzed complex system.

The estimated network measures in terms of node degree and node strength are summarized in Table II for all the analyzed connectivity measures. The results of Table II can be easily correlated to the structure of the modeled system since the network graphs of Fig. 4 are rather simple without high complexity of interactions between the signals. The central role of the activation signal $y_{1}$ is indicated by the high out-degree values estimated by the PDC, rPDC, and DTF measures. In addition, the reachability property of signal $y_{1}$ is evident by the high out-strength value given by the DTF measure. It should

b

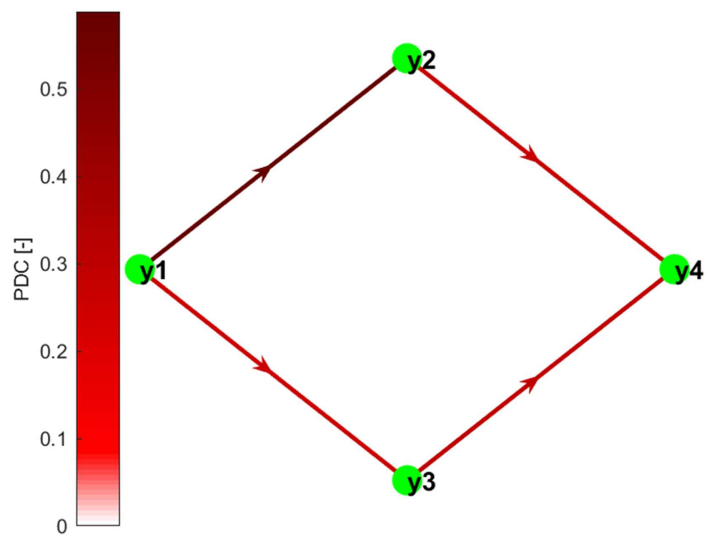

d

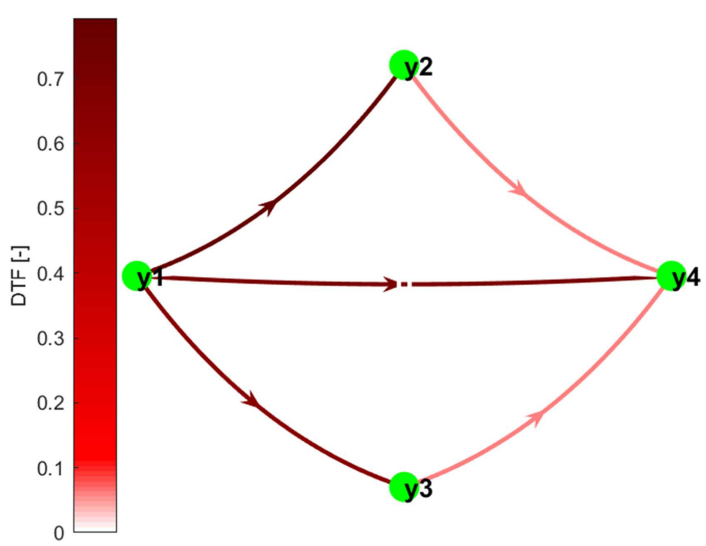

FIG. 3. Connectivity network graphs based on the coherence function (a), PDC (b), rPDC (c), and DTF (d) connectivity measures for the multivariate system (8) at the nominal frequency of $1 \mathrm{~Hz}$. 


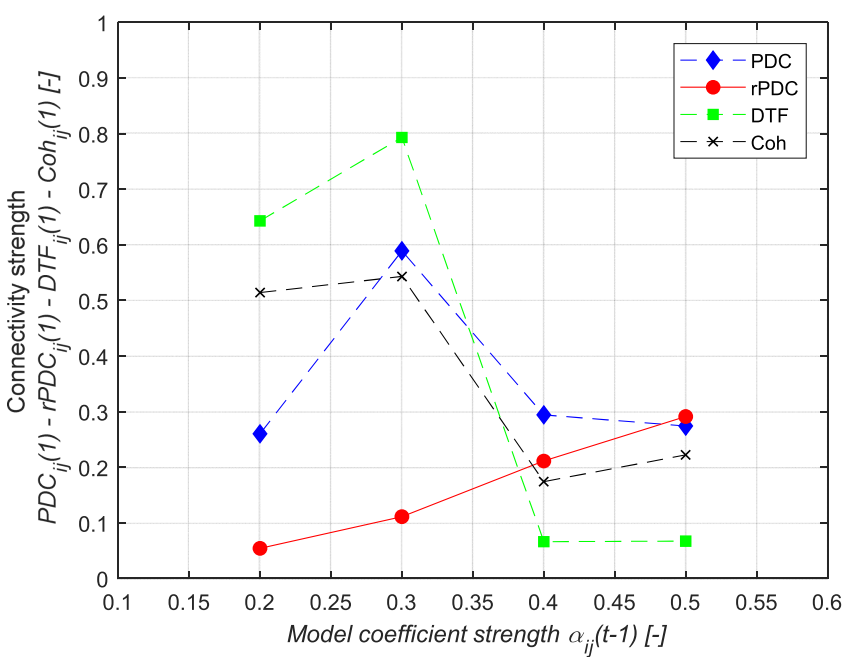

FIG. 4. Connectivity strength values as a function of coefficient values in the MVAR model (8) at the nominal frequency of $1 \mathrm{~Hz}$.

be noted that the coherence function is not differentiating between in- and out-degree/strength, as it is an undirected connectivity measure.

This simple analytical model is demonstrating the advantages, limitations, and difference between the coherence function and the PDC, rPDC, and DTF connectivity measures. Clearly, the rPDC and DTF measures can be used complementary to each other for analyzing the structural behavior of a system and for indicating the possible source of perturbation. Therefore, the connectivity analysis will be focusing solely on rPDC and DTF in the following applications, based on simulated and measured signals of nuclear reactors.

\section{APPLICATION ON NUCLEAR REACTOR SIMULATED SYSTEM}

The connectivity analysis methodology is applied here to a simulated nuclear reactor system, in order to further assess its capabilities on estimating the structural behavior between various thermal-hydraulic and neutronic signals under a perturbation scenario. To this aim, the Swedish Oskarshamn-2 BWR has been modeled, at PSI, with the best-estimate simulation code
SIMULATE-3K (S3K). ${ }^{20} \mathrm{~S} 3 \mathrm{~K}$ is a state-of-the-art code, which couples both the neutronics (i.e., 3D neutron diffusion equation) and thermal-hydraulics (i.e., continuity and energy conservation equations for liquid and vapor phases and one mixture momentum equation) solvers. S3K evaluates the time-dependent reactor behavior and estimates the thermal margins under the occurrence of various transient scenarios (i.e., perturbation of coolant temperature and flow, propagation of steam waves, reactivity initiated accidents, etc.). In this application, a perturbation in the feedwater flow $\left(Q_{F W}\right)$ is imposed on the simulated boiling water reactor (BWR) system. The source of perturbation is a priori known, and therefore, the capability of the connectivity analysis methodology can be verified and confirmed, by studying the propagation of the perturbation throughout the entire system and consequently estimating the interconnections between the key signals. The simulated signals have a length of 746 data points and have been sampled with $8 \mathrm{~Hz}$ sampling frequency. Both the rPDC and the DTF connectivity measures are utilized here to estimate the direct interconnections and the reachability of the main process signals. The activation signal, $Q_{F W}$, is modeled as damped oscillator with a nominal frequency of $1 \mathrm{~Hz}$. A dynamic simulation is performed by imposing the perturbation of the $Q_{F W}$ across the nuclear reactor system. The postprocessing analysis of the simulation results, in both the time and the frequency domain, reveals the main reactor signals, which are affected by this perturbation. The imposed oscillatory behavior of $Q_{F W}$ results in an oscillatory response of the core coolant flow $\left(Q_{C}\right)$, exit liquid flow $\left(Q_{L E}\right)$, and the core pressure drop $\left(P D_{C}\right)$, as shown in Fig. 5(a). In other words, all these process signals are exhibiting a strong spectral peak at the nominal frequency of $1 \mathrm{~Hz}$, at which the perturbation is designed to take place [Fig. 5(b)], and therefore, emphasis is given on analyzing the interconnections between those processes.

The model order is estimated by Akaike's final prediction criterion as $p=2$, and then a MVAR(2) is fitted to the simulated data. The results of the connectivity analysis, in terms of both the rPDC and the DTF measures and the central role of the activation signal $Q_{F W}$ within the analyzed simulated system, are presented in Fig. 6. Concerning rPDC [Fig. 6(a)], it is clearly observed that the $Q_{F W}$ is directly influencing the behavior of all the other signals, which are further interconnected to each other with strong feedback effects. In addition, the DTF network [Fig. 6(b)] reveals the importance of the activation signal on affecting the three other signals both directly and indirectly. In addition, all the direct connections between the $Q_{C}, Q_{L E}$, and $P D_{C}$, showed in the rPDC network [Fig. 6(a)], appear to be very weak

TABLE II. Node degree and strength based on the PDC, rPDC, DTF, and coherence connectivity measures for the MVAR model (8) at a nominal frequency of $1 \mathrm{~Hz}$. The values of the key signals are highlighted with boldface.

\begin{tabular}{|c|c|c|c|c|c|c|c|c|c|c|c|c|c|c|c|c|c|}
\hline \multirow[b]{3}{*}{ Measure } & \multirow[b]{3}{*}{ Node } & \multicolumn{16}{|c|}{ Causality measure } \\
\hline & & \multicolumn{4}{|c|}{ PDC } & \multicolumn{4}{|c|}{$\mathrm{rPDC}$} & \multicolumn{4}{|c|}{ DTF } & \multicolumn{4}{|c|}{ Coh } \\
\hline & & $y_{1}$ & $y_{2}$ & $y_{3}$ & $y_{4}$ & $y_{1}$ & $y_{2}$ & $y_{3}$ & $y_{4}$ & $y_{1}$ & $y_{2}$ & $y_{3}$ & $y_{4}$ & $y_{1}$ & $y_{2}$ & $y_{3}$ & $y_{4}$ \\
\hline \multirow[t]{2}{*}{ Node degree } & Out & 2 & 1 & 1 & 0 & 2 & 1 & 1 & 0 & 3 & 1 & 1 & 0 & 3 & 3 & 3 & 3 \\
\hline & In & $\mathbf{0}$ & 1 & 1 & 2 & $\mathbf{0}$ & 1 & 1 & 2 & $\mathbf{0}$ & 1 & 1 & 3 & & & & \\
\hline \multirow[t]{2}{*}{ Node strength } & Out & 0.85 & 0.27 & 0.29 & 0.00 & 0.17 & 0.29 & 0.21 & 0.00 & 2.14 & 0.07 & 0.07 & 0.00 & 1.48 & 1.35 & 1.27 & 0.82 \\
\hline & In & 0.00 & 0.59 & 0.26 & 0.57 & 0.00 & 0.11 & 0.05 & 0.50 & 0.00 & 0.79 & 0.64 & 0.83 & & & & \\
\hline
\end{tabular}


a
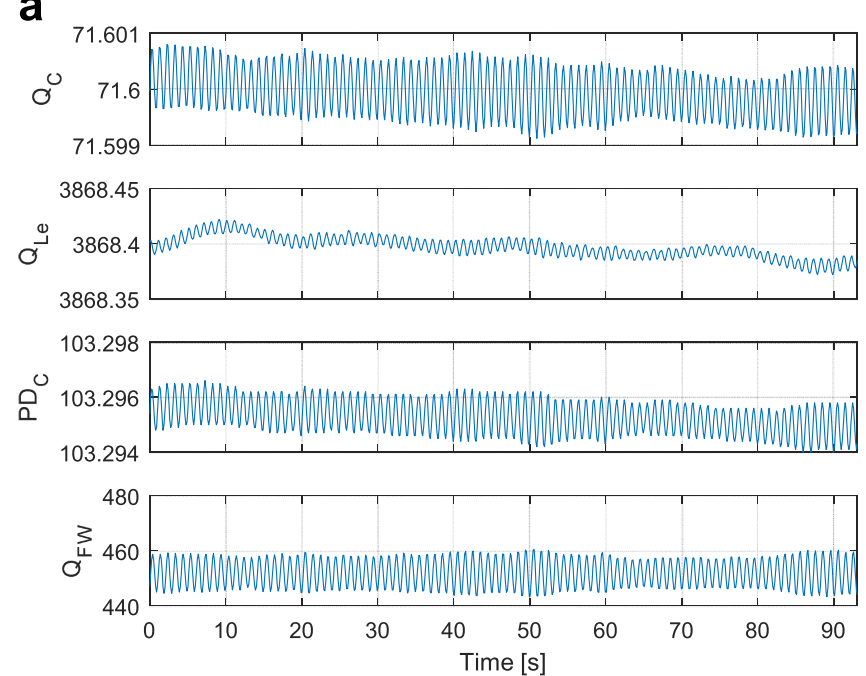

b

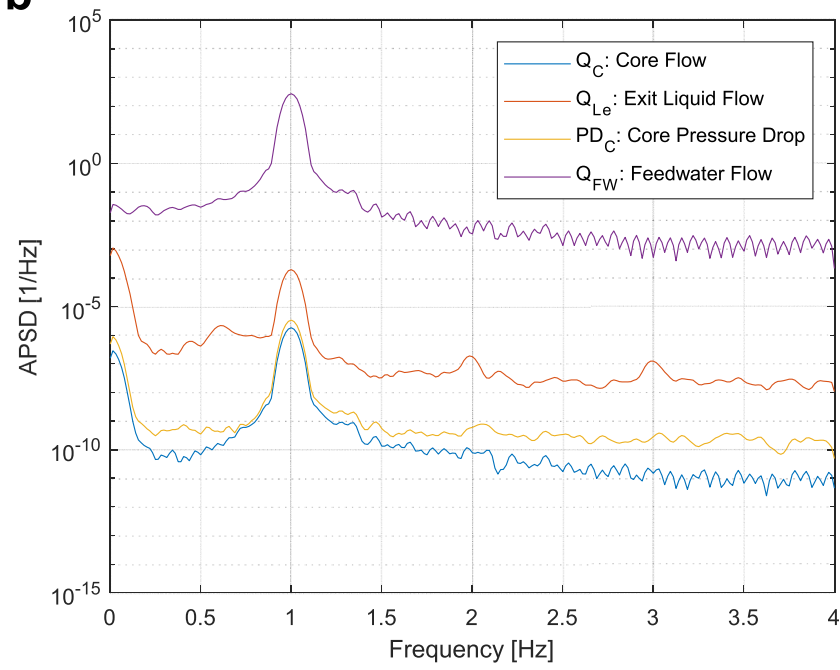

FIG. 5. Simulated nuclear reactor signals in the time domain (a) and the frequency domain (b) in the case of feedwater flow $\left(Q_{F W}\right)$ perturbation at a nominal frequency of $1 \mathrm{~Hz}$.

in the DTF results [Fig. 6(b)]. The DTF analysis is emphasizing on the reachability character of the signals' interrelationships without distinguishing direct from indirect interconnections. Therefore, the interconnections between signals, which are commonly driven by the same activation signal, appear to be very weak in the DTF results. For example, the DTF analysis shows that the $Q_{F W}$ signal is driving both the $Q_{C}$ and the $Q_{L E}$ without revealing any strong interconnection between these two signals.

The importance of the activation signal $Q_{F W}$ can also be identified by the network measures, presented in Table III. The high outdegree values based on $\mathrm{PDC}$ and DTF measures indicate that $Q_{F W}$ is directly affecting the other three signals. Furthermore, it is observed that the out-strength value of $Q_{F W}$, based on both rPDC and DTF

a

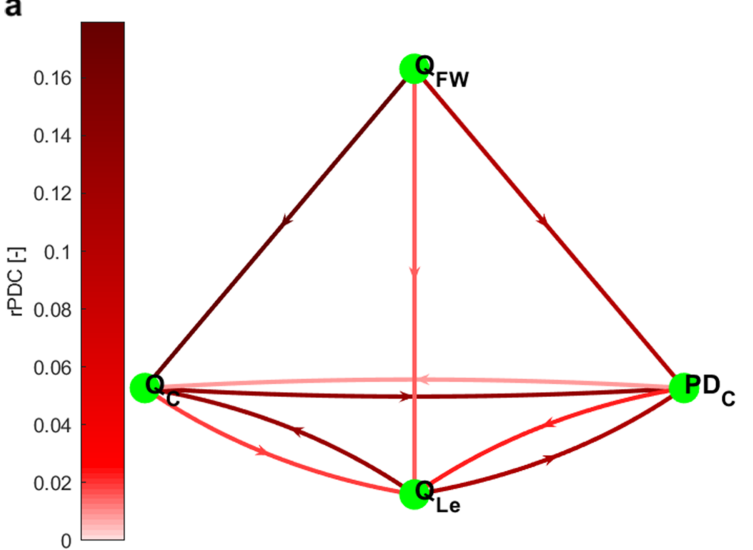

measures, is the highest among all the analyzed signals. This analysis shows that the rPDC and the DTF connectivity measures are both important in causality analysis and shall be used complementary to each other in order to indicate the structural behavior of complex simulated or real systems.

\section{APPLICATION ON BOILING WATER REACTOR PLANT DATA}

After demonstrating the importance of connectivity analysis on predicting the connectivity networks of simulated data, this section is dedicated to more challenging cases, i.e., the analysis of real plant data originating from a Swiss BWR. The advantages and limitations

b

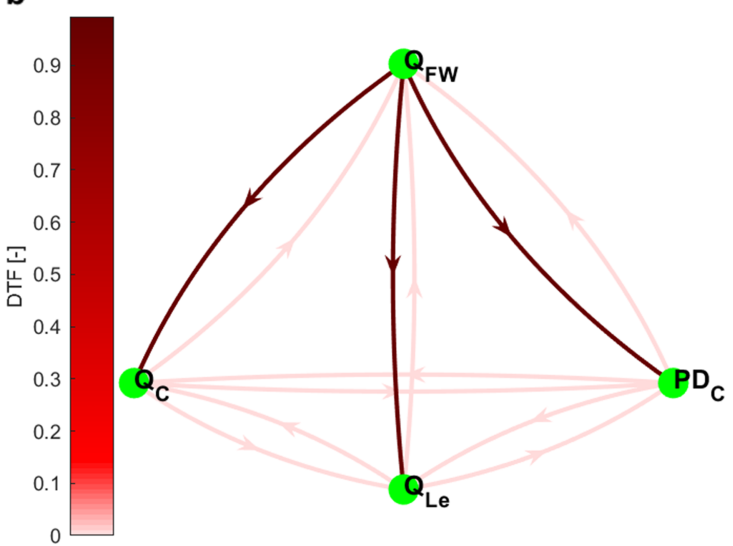

FIG. 6. Connectivity network graphs at $1 \mathrm{~Hz}$ based on the $\operatorname{rPDC}(\mathrm{a})$ and $\mathrm{DTF}(\mathrm{b})$ connectivity measures resulting from a simulated perturbation of the feedwater flow $\left(Q_{F W}\right)$ within a BWR. 
TABLE III. Node degree and strength based on the rPDC and DTF measures for the simulated BWR system at $1 \mathrm{~Hz}$. The values of the key signals are highlighted with boldface.

\begin{tabular}{|c|c|c|c|c|c|c|c|c|c|}
\hline \multirow[b]{3}{*}{ Measure } & \multirow[b]{3}{*}{ Node } & \multicolumn{8}{|c|}{ Causality measure } \\
\hline & & \multicolumn{4}{|c|}{ rPDC } & \multicolumn{4}{|c|}{ DTF } \\
\hline & & $Q_{C}$ & $Q_{L E}$ & $P D_{C}$ & $Q_{F W}$ & $Q_{C}$ & $Q_{L E}$ & $P D_{C}$ & $Q_{F W}$ \\
\hline \multirow[t]{2}{*}{ Node degree } & Out & 2 & 2 & 2 & 3 & 3 & 3 & 3 & 3 \\
\hline & In & 3 & 3 & 3 & $\mathbf{0}$ & 3 & 3 & 3 & 3 \\
\hline \multirow[t]{2}{*}{ Node strength } & Out & 0.15 & 0.24 & 0.03 & 0.30 & 0.05 & 0.03 & 0.05 & 2.98 \\
\hline & In & 0.32 & 0.06 & 0.35 & 0.00 & 0.99 & 0.99 & 0.99 & 0.01 \\
\hline
\end{tabular}

of the rPDC and DTF causality estimators on inferring the causal interactions in highly coupled systems are demonstrated in this section. The first data set was recorded during a stability event that occurred during the start-up operation of the reactor, which led to high decay ratios (DRs) with the corresponding resonance frequency (RF) of about $0.9 \mathrm{~Hz}$ (Sec. V A). The second set corresponds to the start-up conditions during the generator synchronization of different fuel cycles, at low power and flow levels, which led unexpectedly to high decay ratios (Sec. V B).

\section{A. High decay ratio during start-up of KKL cycle $\mathbf{2 4}$}

BWRs are systems susceptible, under certain conditions, to power oscillations leading to instabilities, even if they are designed to be very stable at normal operating conditions. These instabilities are characterized by high complexity due to the tight coupling between neutronic and thermal-hydraulic via mainly the void reactivity feedback. DR is the parameter that quantifies the stability margin, by estimating how rapidly a disturbance within the core is damped. In other words, a reactor is stable when DR is less than 1 , whereas it is unstable (stability event) when DR is greater than 1 . In this section, a stability

a

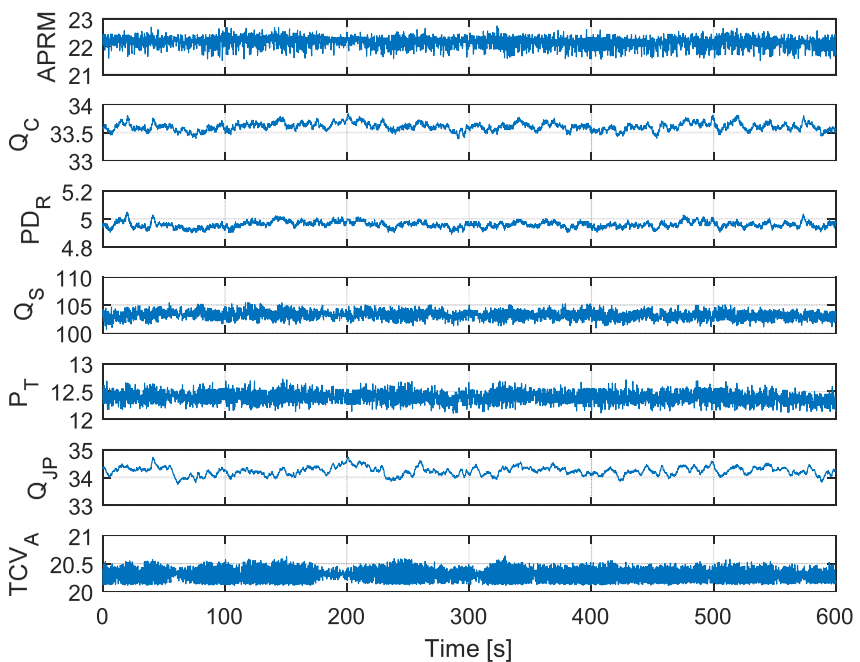

event (DR 1) of the Swiss BWR Leibstadt (KKL), which occurred during the start-up of the 24th operational cycle, is studied. This stability event has been extensively analyzed previously in Ref. 21 using advanced time-series modeling approaches in both the time domain and the frequency domain [i.e., continuous and structural autoregressive moving average (CSARMA) modeling approach, and the signal transmission path (STP) method, respectively]. The analysis in Ref. 21 focused on estimating the interconnectivity between the neutron detector responses (i.e., time-dependent fluctuation of neutron population at various core locations) and the steam flow $\left(Q_{S}\right)$ and reactor pressure signals. It was indicated that the main contributor to the observed high DR was not due to core instability, but it is rather caused by a system disturbance, without any further indication about the precise parameter/component, which triggered the event. In the current work, the analyzed reactor system is extended by including key process signals related to the turbine operation, such as the turbine pressure $\left(P_{T}\right)$ and a turbine control valve $\left(T C V_{A}\right)$. The simultaneously recorded signals have a length of 4800 data points and have been sampled with $8 \mathrm{~Hz}$ sampling frequency. Figure 7 (a) shows the time series of the main nuclear reactor signals (i.e., $A P R M$ : average power range monitor; $Q_{C}$ : core coolant flow; $P D_{R}$ : reactor

b

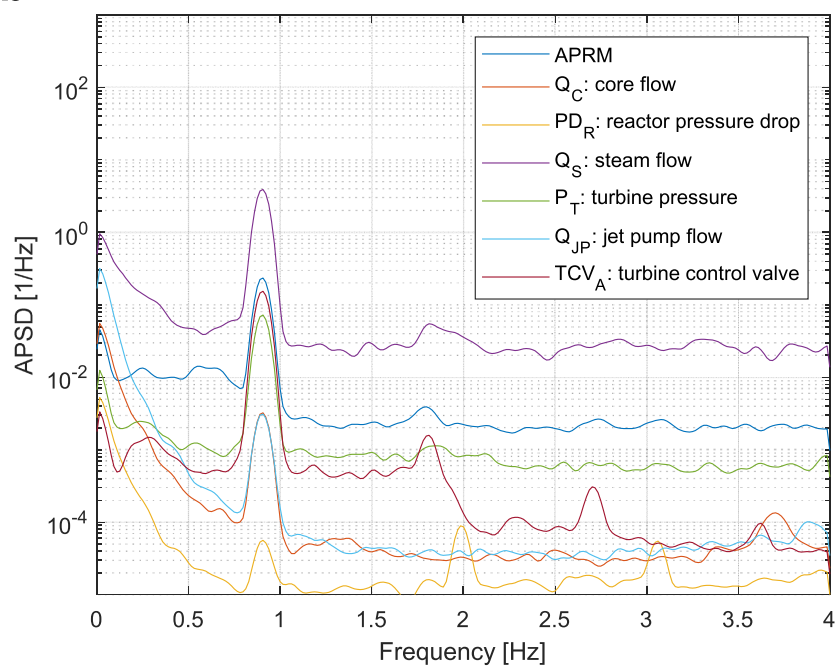

FIG. 7. Nuclear reactor signals during the stability event of KKL cycle 24 , in the time domain (a) and the frequency domain (b). 
a

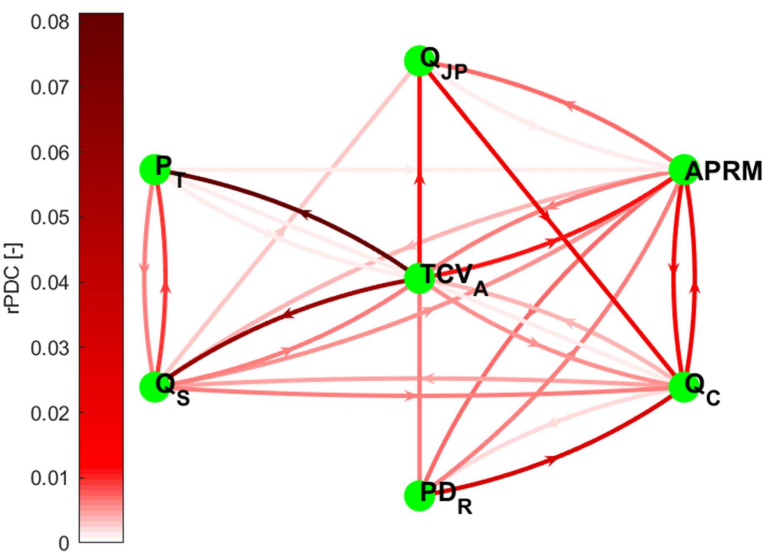

b

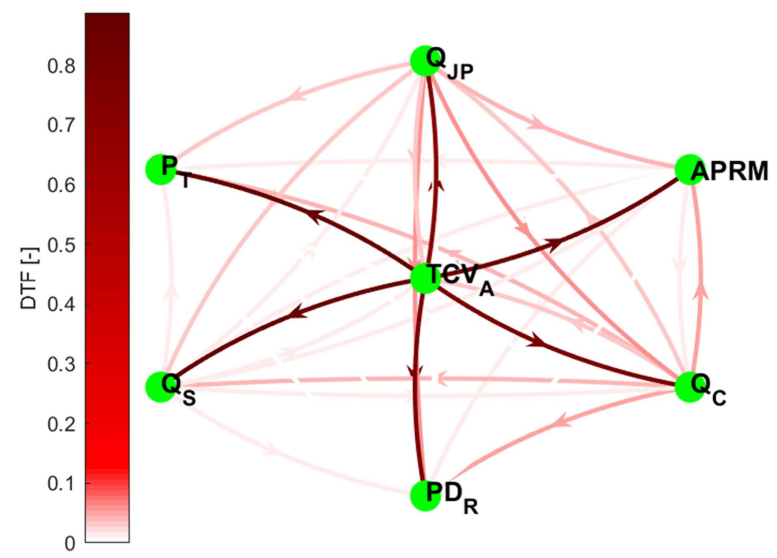

FIG. 8. Connectivity network graphs based on the rPDC (a) and DTF (b) connectivity measures for the stability event of KKL cycle 24 , at a resonance frequency of $0.9 \mathrm{~Hz}$.

pressure drop; $Q_{S}$ : steam flow; $P_{T}$ : turbine pressure; $Q_{J P}$ : jet pump flow; and $T C V_{A}$ ) during the occurrence of the stability event. In addition, the frequency analysis of the examined signals indicates that all of them exhibit a strong spectral peak at the resonance frequency of $0.9 \mathrm{~Hz}$ [Fig. 7(b)].

The model order is estimated by the Bayesian information criterion as $p=5$, and then a MVAR(5) is fitted to the measured data. The connectivity analysis results are summarized in Fig. 8 in terms of the connectivity measures rPDC [Fig. 8(a)] and DTF [Fig. 8(b)]. Both connectivity networks indicate the central role of the $T C V_{A}$ behavior on the analyzed system. First, Fig. 8(a) shows that the oscillatory movement of the opening position of $T C V_{A}$ at $0.9 \mathrm{~Hz}$ is directly driving the response of almost all the other signals. In addition, the DTF network [Fig. 8(b)] demonstrates the reachable character that the $T C V_{A}$ has within the analyzed system during the stability event. In other words, the oscillatory movement of the opening position of this valve is affecting all the other reactor signals leading to the observed stability event. It should be noted that, in Ref. 21 it was shown that the stability event was originated from a disturbance related to the steam lines. While the current analysis justifies, with greater confidence, the conclusion made in Ref. 21, it could additionally, with the help of connectivity analysis, identify precisely the real source of perturbation, i.e., $T C V_{A}$.
The network graphs of this example are rather complex and it might be challenging to interpret properly the structural behavior of the analyzed system. The usefulness of network measures (e.g., node degree and strength) can be demonstrated by such systems in which the interconnections are complex and it is not straightforward to estimate the possible source of perturbation. The results of the network measures based on the rPDC and DTF network graphs are presented in Table IV. The APRM, $Q_{S}$, and $T C V_{A}$ have the highest out-degree values based on the rPDC analysis, whereas all signals apart the $P D_{R}$ and $P_{T}$ are exhibiting the highest possible out-degree with respect to the DTF measure. The central role of the $T C V_{A}$ signal is demonstrated by the out-strength values, estimated by both $\mathrm{rPDC}$ and DTF measures, which are by far greater than any other signal.

\section{B. High decay ratio during start-up of KKL cycle 33}

The second data set to be analyzed in this paper corresponds to low power (18.8\%) and low coolant flow rate (33.5\%) conditions, during the generator synchronization of the KKL core. The systematic analysis of the plant data by using the PSI stability methodology ${ }^{22}$ (not shown here), revealed high decay ratios (i.e., $D R=0.6-0.9$ ) at the very low resonance frequency of $0.27 \mathrm{~Hz}$ for most of the in-core neutron detectors. It is well known in stability analysis, however, that at such low core power/flow levels, the reactor core is expected to

TABLE IV. Node degree and strength based on the rPDC and DTF measures for the stability event of KKL cycle 24 at $0.9 \mathrm{~Hz}$. The values of the key signals are highlighted with boldface.

\begin{tabular}{|c|c|c|c|c|c|c|c|c|c|c|c|c|c|c|c|}
\hline \multirow[b]{3}{*}{ Measure } & \multirow[b]{3}{*}{ Node } & \multicolumn{14}{|c|}{ Causality measures } \\
\hline & & \multicolumn{7}{|c|}{$\mathrm{rPDC}$} & \multicolumn{7}{|c|}{ DTF } \\
\hline & & $A P R M$ & $Q_{C}$ & $P D_{R}$ & $Q_{S}$ & $P_{T}$ & $Q_{J P}$ & $T C V_{A}$ & $A P R M$ & $Q_{C}$ & $P D_{R}$ & $Q_{S}$ & $P_{T}$ & $Q_{J P}$ & $T C V_{A}$ \\
\hline \multirow[t]{2}{*}{ Node degree } & Out & 5 & 4 & 3 & 5 & 4 & 2 & 5 & 6 & 6 & 1 & 6 & 0 & 6 & 6 \\
\hline & In & 6 & 6 & 2 & 4 & 2 & 4 & 4 & 4 & 4 & 5 & 4 & 5 & 5 & 4 \\
\hline \multirow[t]{2}{*}{ Node strength } & Out & 0.04 & 0.03 & 0.04 & 0.03 & 0.01 & 0.02 & 0.17 & 0.09 & 0.27 & 0.00 & 0.10 & 0.00 & 0.27 & 4.91 \\
\hline & In & 0.05 & 0.08 & 0.01 & 0.07 & 0.09 & 0.03 & 0.02 & 0.97 & 0.88 & 0.92 & 0.97 & 1.00 & 0.79 & 0.10 \\
\hline
\end{tabular}


a
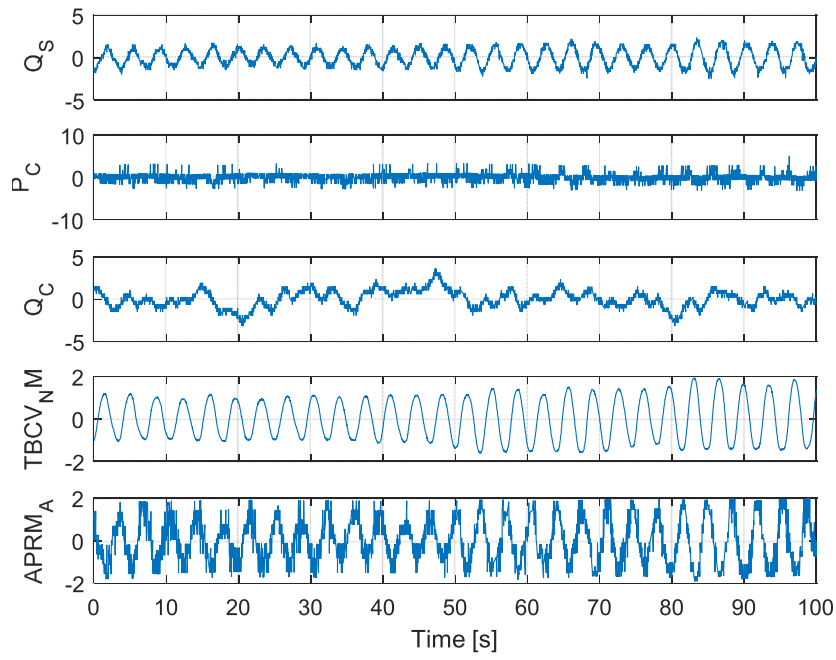

b

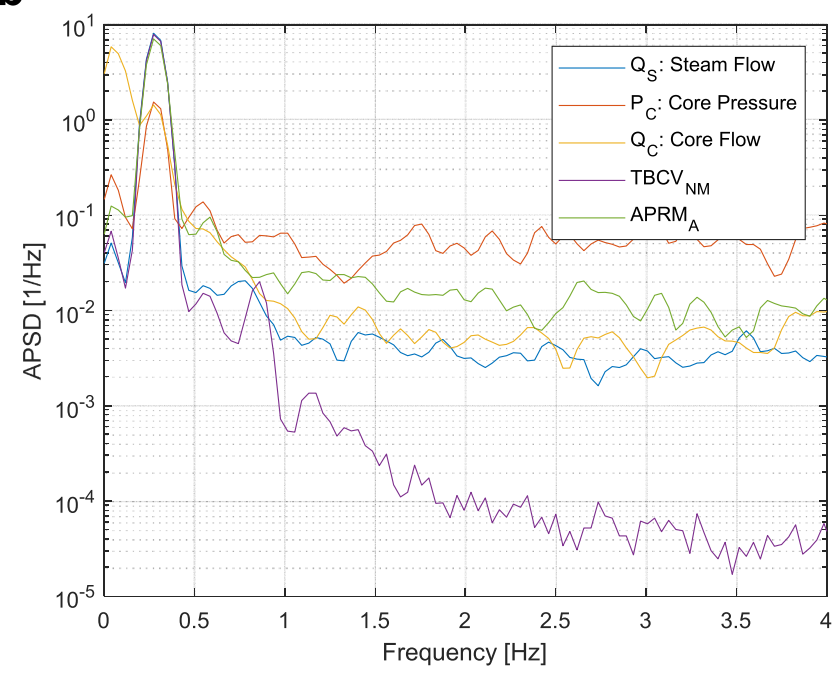

FIG. 9. In-core detector and process signals during the generator synchronization of KKL cycle 33, in the time domain (a) and the frequency domain (b).

be very stable, and therefore, the source of such high decay ratios is intended to be estimated via connectivity analysis. The extended statistical and postprocessing analysis of the measured data in both the time domain and the frequency domain, which is based on the consolidated PSI methodology, ${ }^{22}$ revealed which signals are exhibiting a distinct oscillatory behavior during this measurement. Figure 9 presents the time- and frequency-dependent behavior of these reactor signals: steam flow $\left(Q_{S}\right)$, core pressure $\left(P_{C}\right)$, core coolant flow $\left(Q_{C}\right)$, turbine bypass control valve $\left(T B C V_{N M}\right)$, and average power range monitor $\left(A P R M_{A}\right)$. All signals have a sampling frequency of $8 \mathrm{~Hz}$ and have been recorded over $100 \mathrm{~s}$. These signals, and, in particular, the $Q_{S}, P_{C}$, and $T B C V_{N M}$, exhibit a clear oscillatory behavior at the resonance frequency of $0.27 \mathrm{~Hz}$, with an almost noise-free

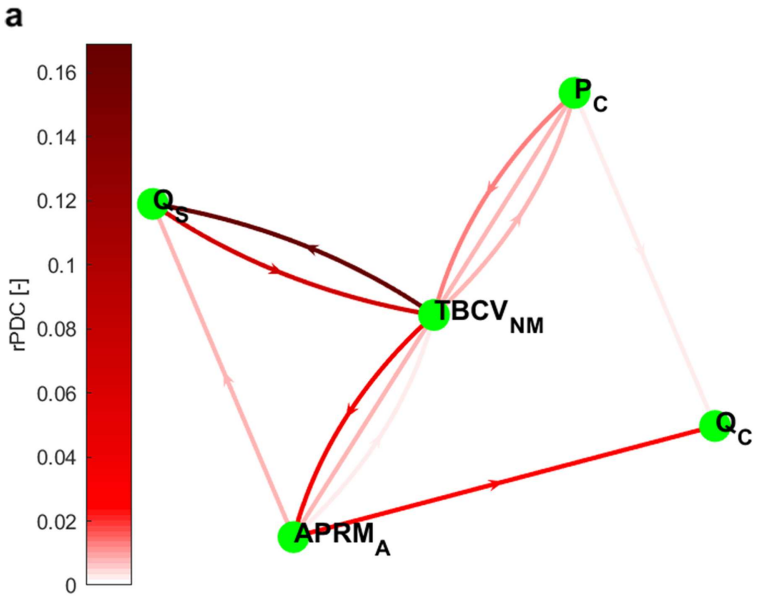

response (nearly pure sine wave) of the opening position of the turbine bypass control valve $T B C V_{N M}$.

For this example, the model order is estimated by the Bayesian information criterion as $p=6$, and then a $\operatorname{MVAR}(6)$ is fitted to the measured data. Figure 10 represents the network graphs originating from the rPDC [Fig. 10(a)] and the DTF [Fig. 10(b)] connectivity measures. The $T B C V_{N M}$ signal clearly drives the response of both the steam flow and the neutron signal, based on the rPDC analysis. In addition, the bypass control valve fluctuation is evidently propagating throughout the entire system, based on the DTF analysis, indicating its essential impact during the synchronization of the generator. The key role of the $T B C V$ can be explained by the fact that throughout the generator synchronization the turbine control valves (TCVs) b

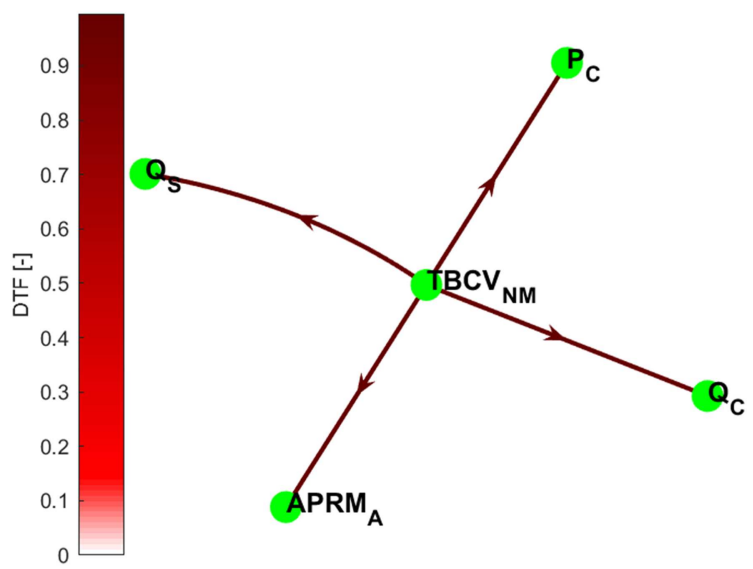

FIG. 10. Connectivity network graphs based on the rPDC (a) and DTF (b) connectivity measures during the start-up of KKL cycle 33 , at the resonance frequency of $0.27 \mathrm{~Hz}$. 
TABLE V. Node degree and strength based on the PPDC and DTF measures during the start-up of KKL cycle 33. The values of the key signals are highlighted with boldface.

\begin{tabular}{|c|c|c|c|c|c|c|c|c|c|c|c|}
\hline \multirow[b]{3}{*}{ Measure } & \multirow[b]{3}{*}{ Node } & \multicolumn{10}{|c|}{ Causality measures } \\
\hline & & \multicolumn{5}{|c|}{ rPDC } & \multicolumn{5}{|c|}{ DTF } \\
\hline & & $Q_{s}$ & $P_{C}$ & $Q_{C}$ & $T B C V_{N M}$ & $A P R M_{A}$ & $Q_{S}$ & $P_{C}$ & $Q_{C}$ & $T B C V_{N M}$ & $A P R M_{A}$ \\
\hline \multirow[t]{2}{*}{ Node degree } & Out & 1 & 3 & 0 & 3 & 3 & 1 & 1 & 0 & 4 & 0 \\
\hline & In & 2 & 1 & 2 & 3 & 2 & 1 & 1 & 1 & 0 & 1 \\
\hline \multirow[t]{2}{*}{ Node strength } & Out & 0.07 & 0.02 & 0.00 & 0.22 & 0.04 & 0.00 & 0.00 & 0.00 & 3.98 & 0.00 \\
\hline & In & 0.18 & 0.01 & 0.03 & 0.09 & 0.05 & 1.00 & 1.00 & 0.99 & 0.00 & 1.00 \\
\hline
\end{tabular}

are only partially open as the steam is dumped to the condenser. During the analyzed operation stage, the opening positions of the TBCVs are adjusted based on the steam dome pressure control system until the generator is synchronized and the steam is directed to the TCVs. Therefore, when the steam dome pressure exceeds certain limits, then the $T B C V$ positions are opening and vice-versa [this is why an interconnection it is observed from $P_{c}$ to TBCV in Fig. 10(a)]. Consequently, the TBCVs exhibit the oscillatory behavior, as depicted in Fig. 9(a), and interfere with all the other reactor processes as the connectivity networks indicated in Fig. 10.

Table $\mathrm{V}$ is summarizing the results of network measures for the second data set. On the one hand, the rPDC analysis indicates the driving behavior that the $P_{C}, T B C V_{N M}$, and $A P R M_{A}$ have by exhibiting high out-degree values. On the other hand, the DTF results demonstrate that all the observed interconnections are driven by the $T B C V_{N M}$ behavior, which has the maximum possible out-degree value. It should be noted that the $T B V C_{N M}$ shows the highest outstrength value based on both rPDC and DTF analyses. All these indicate that the turbine bypass valve should have a central role within the analyzed system and its behavior can be responsible for the observed perturbation during the generator synchronization. Clearly, even in such a complex system, as a BWR, the connectivity analysis can indicate a possible source of perturbations.

\section{CONCLUSIONS}

Causality analysis is a valuable tool for studying the interdependencies between signals and for extracting information about their cause-and-effect relationships. In this paper, the connectivity analysis principles are applied in the field of nuclear reactors. A nuclear reactor is a highly complex system, characterized by strong coupling mechanisms between most of its processes. The deeper understanding of the structural behavior of nuclear processes and the identification of root cause of potential anomalies and/or disturbances provide key information that can assist on taking the appropriate actions for the safe operation of the nuclear reactor. The advantage of this methodology is the fast and robust evaluation of signals connectivity by using multivariate autoregressive advanced signal processing techniques and connectivity measures. The rPDC measure identifies the direct directional connections between signals and estimates the strength of the analyzed causal interactions. The DTF measure is used as a valuable complementary connectivity measure for indicating the signals' reachability within the analyzed system. As a first step, both measures have been tested and correctly identified the activation signals, i.e., source of perturbation, in a simple analytical model and in a simulated nuclear reactor system. After that, the connectivity analysis methodology has been used for analyzing two real plant data sets, originating from the Swiss BWR, KKL. The first data set corresponds to a stability event that took place during the start-up phase of the 24th cycle, leading to power oscillations with high decay ratios at a frequency of $0.9 \mathrm{~Hz}$. The connectivity analysis indicates clearly that the source of distortion is related to the perturbation of the opening position of a turbine control valve. This signal has a clear oscillatory behavior at the nominal frequency of $0.9 \mathrm{~Hz}$ during the occurrence of the stability event. The rPDC connectivity measure of this signal revealed the interconnections with the main reactor signals (i.e., neutron detectors, core coolant and steam flow, reactor pressure drop, jet pump flow, etc.), indicating that the event occurred due a control system disturbance and not induced by the core instability. The second analyzed data set was recorded during the generator synchronization phase, at the start-up of another operating cycle of the same BWR. Analysis of these data revealed unexpectedly high decay ratio values at a frequency of $0.27 \mathrm{~Hz}$ at low power and low core coolant flow operating conditions. Results showed, clearly, the central role of the bypass control valves in affecting the reactor system through the estimation of the rPDC and DTF measures. It can be concluded, therefore, that the connectivity analysis has been successfully applied, for the first time, in the nuclear reactor field, and has led to a comprehensive study allowing the identification of the relationships between different processes, and more importantly, estimating the causes triggering certain disturbances or anomalies in nuclear reactors.

\section{ACKNOWLEDGMENTS}

This work is part of a Swiss Federal Institute of Technology at Lausanne (EPFL) Ph.D. thesis on neutron noise modeling and partially funded by swissnuclear, the nuclear energy section of the Swiss electricity companies. The authors would like to acknowledge Dr. C. Aguirre and Dr. R. Bieli (Leibstadt nuclear power plant$\mathrm{KKL}$ ) for their support with respect to the KKL measured data and Dr. A. Vasiliev (PSI) for his valuable comments regarding this manuscript.

\section{APPENDIX A: NUCLEAR REACTOR SYSTEM}

\section{Description of the system}

The current section is providing a brief description of a nuclear reactor system's basic principle in order to familiarize the reader with 


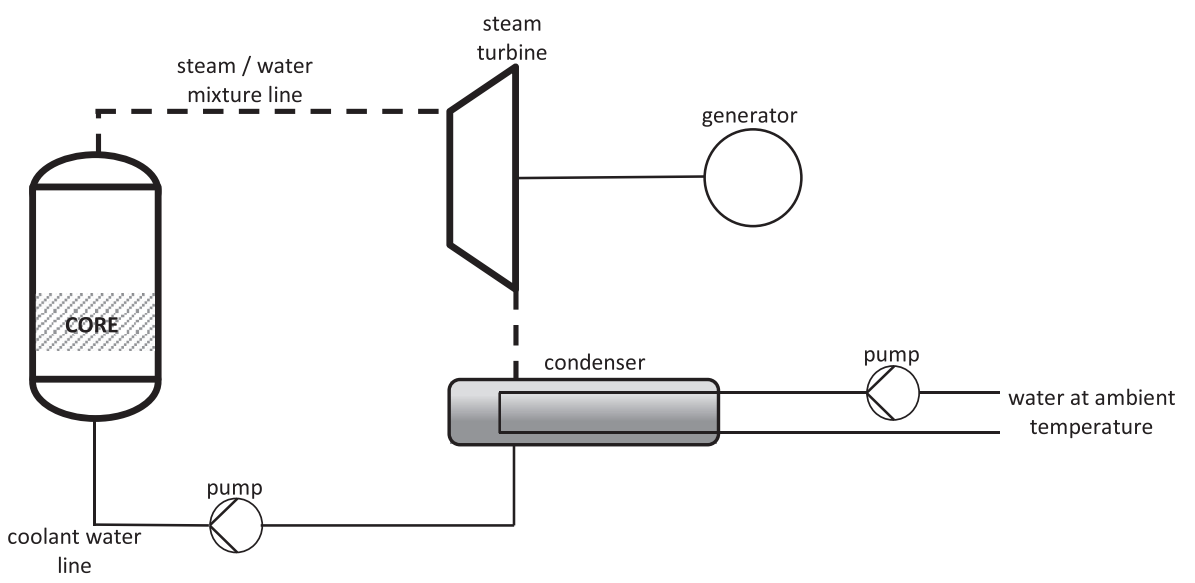

FIG. 11. Simplified schematic of a BWR system.

the specificities of the analyzed system. There are 448 operational nuclear reactors in 30 countries with a net installed capacity of $391 \mathrm{GW}_{\mathrm{e}}$ which supplied $2503 \mathrm{TW}_{\mathrm{e}}$ h of electricity in 2017 (equivalent to the $11 \%$ of the worldwide electricity supply). ${ }^{23}$ The current paper is focusing on the so-called boiling water reactor (BWR) type, which is the second most common nuclear reactor design, and it is widely used in the USA, Japan, Germany, Finland, India, Mexico, Spain, Sweden, and Switzerland. Figure 11 illustrates in a schematic representation the simplified operation of a BWR. A BWR is generating heat by nuclear fission reactions, taking place inside the nuclear reactor core. A nuclear fission reaction occurs when a neutron is absorbed by a $U^{235}$-nucleus, which will subsequently split into two daughter nuclei by releasing two to three new neutrons and by producing heat. The newly released neutrons will be then absorbed by other $\mathrm{U}^{235}$-nuclei and further nuclear fission reactions will take place and more heat will be produced. $U^{235}$ is an isotope of uranium, which is used in the form of uranium dioxide as a nuclear fuel. The uranium dioxide is compressed into small pellets (cylindrical shape of $\sim 1 \mathrm{~cm}$ height and $\sim 1 \mathrm{~cm}$ diameter), being stacked into hosting tubes (i.e., cladding tubes of $\sim 3.5 \mathrm{~m}$ height) and they consist of the so-called fuel pins. As an example, a set of 100 fuel pins in an arrangement of $10 \times 10$ are defining a fuel assembly (with a typical dimensions of $4 \mathrm{~m}$ height, $15 \mathrm{~cm}$ side size, and $300 \mathrm{~kg}$ weight). More than 600 fuel assemblies could be positioned the one next to each other in a circular configuration and they constitute the so-called nuclear reactor core, which typically could have an installed capacity of more than $1 \mathrm{GW}_{\mathrm{e}}$. The reactor core is positioned within the reactor pressure vessel (RPV), which is a thick-walled cylindrical structure made of steel being able to withstand the high pressure, developed internally due to the core operation. Water (acting as coolant) is pumped in to the RPV through the recirculation pumps in order to remove the generated heat (due to nuclear fission) from the surface of the fuel assemblies and, consequently, to prevent the overheating and possible damage of the nuclear fuel. The water enters and exits the RPV with a typical inlet temperature of $275^{\circ} \mathrm{C}$ and $285^{\circ} \mathrm{C}$, respectively. This $10^{\circ} \mathrm{C}$ water temperature increase due to the heat removal from the fuel surface is sufficient for evaporating the water and generating steam. The water-steam mixture is directed through the steam lines to the steam turbine. The high kinetic energy of the steam turns the blades of the turbine which then rotates the generator and finally electricity can be produced. The steam after the turbine is dumbed to the condenser, acting as a heat exchanger (using water at ambient temperature, coming from a river or from a cooling tower, as a second fluid), and condenses the steam into water which is cooled down, and, thereafter, it is pumped back to the RPV. The water follows a closed cycle and any radioactive elements are ensured to be contained within this cycle without having the risk of any radiation release to the environment.

\section{Monitoring of physical processes}

The safe operation of a nuclear reactor is monitored by a series of detectors, sensors, and gauges. Dedicated data acquisition systems are installed in every reactor, and a large set of signals are simultaneously recorded with a sampling frequency of around $100 \mathrm{~Hz}$ which is sufficient for capturing all the frequency components of the reactor signals. ${ }^{24}$ First, the three-dimensional distribution rate of neutrons throughout the core is measured using the so-called neutron detectors (the abbreviation APRM is used in Sec. V). The knowledge of the time-dependent evolution of the neutron population is of key importance for a nuclear reactor system since the neutrons' generation rate due to nuclear fission reactions defines the generated heat and consequently the reactor power. Several neutron detectors are located at different axial and radial positions inside the reactor core in order to measure with high resolution the time-dependent evolution of the neutron field. The sufficient heat removal from the nuclear fuel surface to the water is achieved by appropriately controlling the water inlet flow to the core (the abbreviation $Q_{C}$ is used in Secs. IV and V). Flow meter devices are installed in the water line for the continuous monitoring of the water inlet core flow. The monitoring of the water flow $\left(Q_{L E}\right)$ and the generated steam flow $\left(Q_{S}\right)$ at the exit of the reactor core is of equal importance since the efficiency of the heat removal process can be assessed. To this aim, flow meter devices are located at the exit of the RPV and at the steam/water mixture line. Finally, the pressure of the steam that enters the steam turbine $\left(P_{T}\right)$ is continuously monitoring from appropriate gauges for estimating the pressure losses across the steam lines and the safety margin from the critical operation of the turbine. The importance of all the other 
processes that have been analyzed throughout this paper can be given in a similar manner. More information about the physical processes that are involved in the nuclear reactor operation can be found in the literature. ${ }^{2}$

This brief discerption of the nuclear reactor system is clearly indicating the highly coupling relationship between all the analyzed signals. Therefore, the occurrence of a perturbation scenario, due to the deviation of a single or set processes from their normal operation, may lead to the disturbance of many other parameters. With the help of connectivity analysis, it is intended to identify the root cause and the connectivity pattern between the underlying processes.

\section{APPENDIX B: FREQUENCY DEPENDENCY OF CONNECTIVITY MEASURES AND RESPECTIVE MODEL COEFFICIENTS}

The connectivity network graphs, presented in the current paper (i.e., Figs. 3, 4, 6, 8, and 10), provide the results of connectivity measures on the respective analyzed system at a specific nominal frequency of interest. In addition, the current section is presenting the frequency dependency of the connectivity measures for two representative cases: (1) the analytical model and (2) the measured system of KKL cycle 33, introduced in Secs. III and V B, respectively.
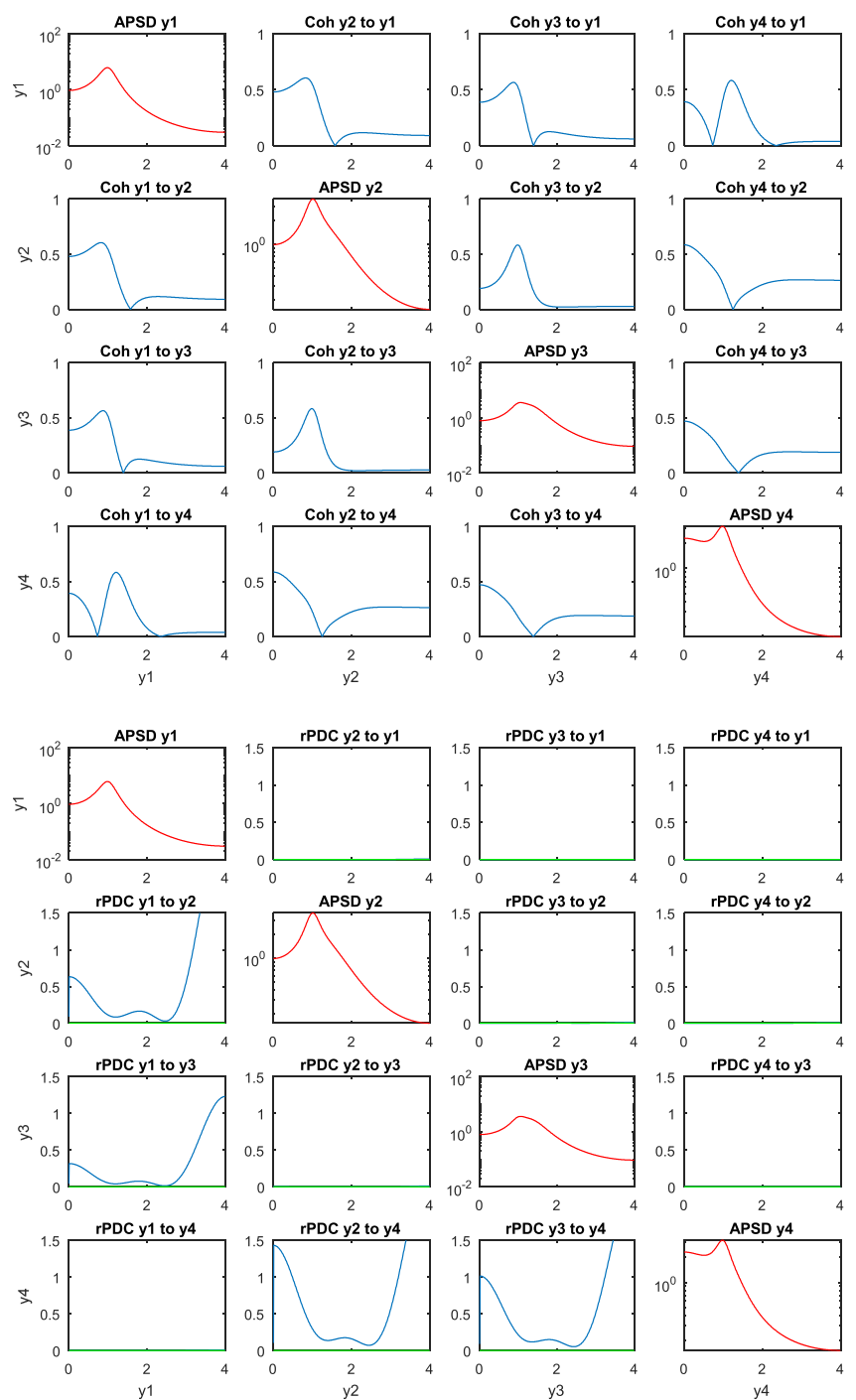
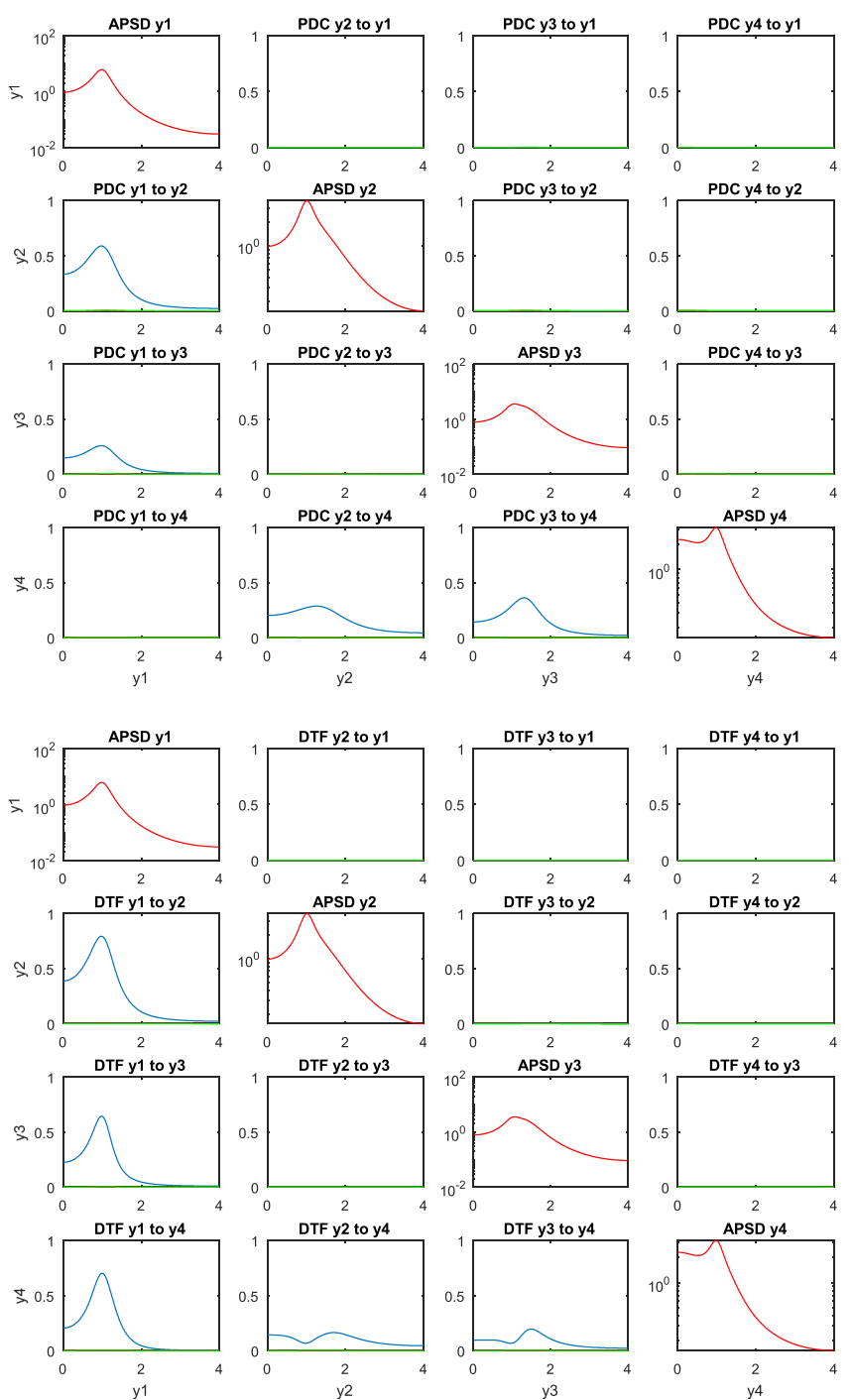

FIG. 12. Frequency dependency of the connectivity measures of the analytical model (Sec. III). 


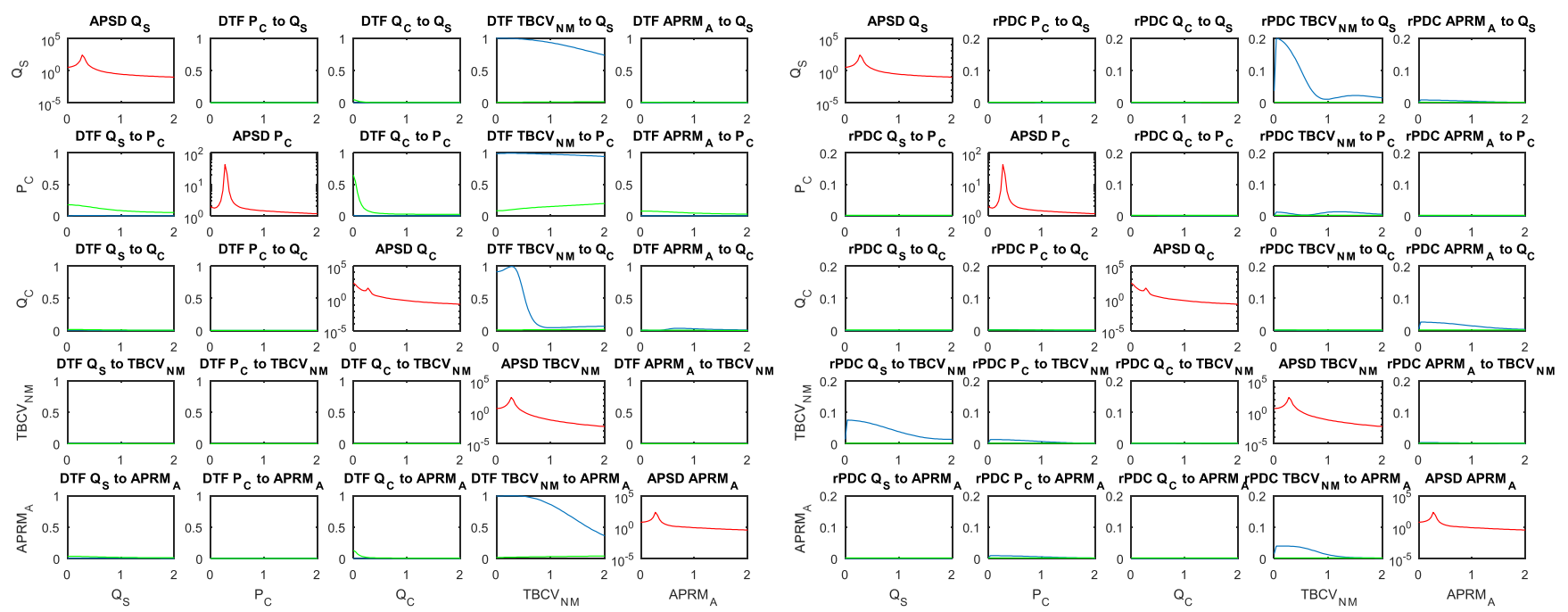

FIG. 13. Frequency dependency of the connectivity measures of the measured data set from KKL cycle 33 (Sec. V B).

\section{The analytical model}

The modeling of the equation system (8) with a MVAR(2) results to the following model coefficients $\boldsymbol{\alpha}(k)$ :

$$
\begin{aligned}
\boldsymbol{a}_{i j}(1)= & {\left[\begin{array}{cccc}
1.19 & 0.02 & -10^{-3} & 10^{-3} \\
-0.42 & 0.50 & 0.01 & 0.02 \\
-0.28 & 3 \cdot 10^{-3} & 0.69 & 10^{-3} \\
0.01 & 0.39 & 0.33 & 0.49
\end{array}\right], } \\
\boldsymbol{a}_{i j}(2)= & {\left[\begin{array}{cccc}
0.70 & -0.003 & 10^{-3} & -3 \cdot 10^{-4} \\
0.01 & -0.30 & 0.01 & 6 \cdot 10^{-3} \\
0.002 & 0.02 & -0.50 & -10^{-5} \\
-0.003 & 0.07 & 0.003 & 0.02
\end{array}\right] . }
\end{aligned}
$$

The frequency dependency of the results of connectivity measures is presented in Fig. 12.

\section{The measured system of KKL cycle 33}

The modeling of the measured data set from KKL cycle 33 (Sec. V B) with a MVAR(6) results to the following model coefficients $\boldsymbol{\alpha}(k)$ :

$$
\begin{aligned}
\boldsymbol{a}_{i j}(1)= & {\left[\begin{array}{ccccc}
0.260 & 0.001 & -0.010 & -0.400 & -0.007 \\
-0.010 & 0.187 & -0.024 & -0.126 & -0.066 \\
0.005 & 0.004 & 0.437 & 0.060 & 0.021 \\
0.001 & 10^{-5} & 0.002 & 0.816 & -0.001 \\
-0.021 & -0.003 & 0.02 & -0.313 & 0.288
\end{array}\right], } \\
\boldsymbol{a}_{i j}(2)= & {\left[\begin{array}{ccccc}
0.169 & -0.002 & -0.010 & 0.094 & -0.009 \\
-0.040 & 0.025 & 0.046 & 0.804 & 0.029 \\
0.023 & -0.004 & 0.135 & -0.122 & 0.011 \\
-0.004 & 10^{-5} & 0.001 & 0.299 & -0.001 \\
-0.023 & -0.005 & -0.009 & -0.430 & 0.167
\end{array}\right], }
\end{aligned}
$$

$$
\begin{aligned}
\boldsymbol{a}_{i j}(3)= & {\left[\begin{array}{ccccc}
0.028 & 0.006 & 0.017 & 0.201 & 0.004 \\
-0.097 & 0.026 & 0.040 & 0.603 & -0.040 \\
-0.011 & -0.007 & 0.058 & 0.194 & 0.005 \\
-0.004 & 10^{-5} & 10^{-5} & 0.127 & 0.001 \\
-0.012 & -0.003 & -0.017 & 0.503 & 0.081
\end{array}\right], } \\
\boldsymbol{a}_{i j}(4)= & {\left[\begin{array}{ccccc}
0.08 & -0.005 & -0.022 & -0.168 & -0.013 \\
-0.03 & 0.016 & -0.030 & -0.438 & 0.020 \\
0.007 & -0.001 & 0.093 & -0.314 & 0.015 \\
0.001 & 0.001 & 0.001 & 0.036 & -0.001 \\
0.02 & -0.013 & 0.026 & -0.074 & 0.081
\end{array}\right], } \\
\boldsymbol{a}_{i j}(5)= & {\left[\begin{array}{ccccc}
0.118 & -0.001 & -0.005 & 0.136 & -9 \cdot 10^{-4} \\
0.013 & 0.012 & 0.033 & 0.870 & -0.024 \\
0.002 & 0.009 & 0.130 & 0.044 & 0.012 \\
-0.008 & 0.001 & 10^{-5} & -0.086 & -0.001 \\
-0.045 & 0.004 & -0.007 & 0.389 & 0.063
\end{array}\right], } \\
\boldsymbol{a}_{i j}(6)= & {\left[\begin{array}{ccccc}
0.065 & -10^{-3} & 0.026 & 0.387 & -0.012 \\
0.067 & 0.028 & -0.033 & -1.819 & 0.026 \\
-0.017 & 0.007 & 0.127 & 0.157 & 0.017 \\
-0.006 & 0.001 & -0.001 & -0.189 & -0.001 \\
-0.038 & -0.010 & -0.010 & -0.348 & 0.025
\end{array}\right] . }
\end{aligned}
$$

The frequency dependency of the results of connectivity measures are presented in Fig. 13.

\section{REFERENCES}

${ }^{1}$ C. Demazière et al., presented at ANS Physor Conference, Cancun, Mexico, 22-26 April 2018.

${ }^{2}$ M. Kaminski and H. Liang, Crit. Rev. Biomed. Eng. 33, 4 (2005).

${ }^{3}$ L. Faes et al., IEEE Trans. Biomed. Eng. 51, 7 (2004).

${ }^{4}$ C. Granger, Econometrica 37, 424-438 (1969).

5 J. Geweke, J. Am. Stat. Assoc. 77, 304 (1982).

${ }^{6}$ Y. Chen, S. Bressler, and M. Ding, J. Neurosci. Methods 150, 228-237 (2006). 
${ }^{7}$ L. Marple, Digital Spectral Analysis with Applications (Prentice-Hall, Inc., Upper Saddle River, NJ, 1987)

${ }^{8}$ A. Schlögl, Signal Process. 86, 9 (2006).

${ }^{9} \mathrm{M}$. Rubinov and O. Sporns, Neuroimage 52, 3 (2010).

${ }^{10} \mathrm{H}$. Elsegai et al., J. Neurosci. Methods 245, 91-106 (2015).

${ }^{11}$ L. Baccalá and K. Sameshima, Biol. Cybern. 84, 6 (2001).

${ }^{12}$ B. Schelter et al., J. Neurosci. Methods 152, 1-2 (2006).

${ }^{13}$ B. Schelter et al., J. Neurosci. Methods 179, 1 (2009).

${ }^{14} \mathrm{M}$. Kaminski and K. Blinowska, Biol. Cybern. 65, 3 (1991).

${ }^{15}$ L. Baccalá, D. Takahashi, and K. Sameshima, IEEE Trans. Biomed. Eng. 63, 12 (2016).
${ }^{16}$ M. Eichler, Biol. Cybern. 94, 6 (2006).

${ }^{17} \mathrm{~J}$. Gross, Graph Theory and Its Applications (Chapman and Hall/CRC, 1999).

${ }^{18}$ E. Bullmore and O. Sporns, Nat. Rev. Neurosci. 10, 186-198 (2009).

${ }^{19}$ A. Delorme et al., Comput. Intell. Neurosci. 2011, 130714 (2011).

${ }^{20}$ A. Dokhane, H. Ferroukhi, and A. Pautz, Ann. Nucl. Energy 96, 344-353 (2016).

${ }^{21}$ M. Demeshko et al., Ann. Nucl. Energy 75, 645-657 (2015).

22 A. Dokhane et al., Ann. Nucl. Energy 33, 1329-1338 (2006).

${ }^{23}$ International Atomic Energy Agency, Nuclear Power Reactors in the World (IAEA, 2018).

${ }^{24} \mathrm{~J}$. Thie, Power Reactor Noise (American Nuclear Society, 1981).

${ }^{25}$ S. Glasstone and A. Sesonske, Nuclear Reactor Engineering (Springer, 1994). 